Draft VERSion July 19, 2016

Preprint typeset using $\mathrm{IAT}_{\mathrm{E}} \mathrm{X}$ style emulateapj v. 5/2/11

\title{
FARADAY ROTATION MEASURE SYNTHESIS OF INTERMEDIATE REDSHIFT QUASARS AS A PROBE OF INTERVENING MATTER
}

\author{
Kwang Seong Kim ${ }^{1}$, Simon J. Lilly ${ }^{1}$, Francesco Miniati ${ }^{1}$, Martin L. Bernet ${ }^{1}$, Rainer Beck ${ }^{2}$, Shane P. \\ O'Sullivan ${ }^{34}$, and Bryan M. Gaensler ${ }^{54}$ \\ Draft version July 19, 2016
}

\begin{abstract}
There is evidence that magnetized material along the line of sight to distant quasars is detectable in the polarization properties of the background sources. The polarization properties appear to be correlated with the presence of intervening MgII absorption, which is thought to arise in outflowing material from star forming galaxies. In order to investigate this further, we have obtained high spectral resolution polarization measurements, with the VLA and ATCA, of a set of 49 unresolved quasars for which we have high quality optical spectra. These enable us to produce a Faraday Depth spectrum for each source, using Rotation Measure Synthesis. Our new independent radio data confirms that interveners are strongly associated with depolarization. We characterize the complexity of the Faraday Depth spectrum using a number of parameters and show how these are related, or not, to the depolarization and to the presence of $\mathrm{MgII}$ absorption along the line of sight. We argue that complexity and structure in the Faraday Depth distribution likely arise from both intervening material and intrinsically to the background source and attempt to separate these. We find that the strong radio depolarization effects associated with intervening material at redshifts out to $z \approx 1$ arise from inhomogeneous Faraday screens producing a dispersion in Rotation Measure across individual sources of around $10 \mathrm{rad} / \mathrm{m}^{2}$. This is likely produced by disordered fields with strengths of at least $3 \mu \mathrm{G}$.

Subject headings: techniques: polarimetric - galaxies: magnetic fields - radio continuum: galaxies quasars: absorption lines
\end{abstract}

\section{INTRODUCTION}

Cosmic magnetic fields are difficult to observe and difficult to treat theoretically, either analytically or numerically. Consequently fields on the scale of galaxies and larger are still poorly understood and therefore often ignored in the context of the formation and evolution of galaxies. However, detections of magnetic fields in a broad range of astrophysical objects have provided indications for the ubiquitous nature of magnetic fields in the Universe.

Over the last few years, we have developed a line of research aimed at detecting and characterizing magnetized material in and around normal galaxies at high redshift. The approach has been to study compact radio-loud quasars and to search for correlations between the presence of intervening strong MgII absorption in the optical spectra and the polarization properties of the quasars, using the Faraday Rotation. Faraday Rotation describes the rotation of the plane of polarization when polarized electromagnetic radiation traverses a region containing a magnetized plasma.

The amount of rotation depends linearly on the square of the wavelength $\lambda^{2}$. The Rotation Measure (RM) is

kwangseong.kim@phys.ethz.ch

${ }^{1}$ Physics Department, ETH Zurich, Wolfgang-Pauli-Strasse 27, 8093 Zurich, Switzerland

2 Max-Planck-Institut für Radioastronomie, Auf dem Hügel 69, 53121 Bonn, Germany

3 Instituto de Astronomía, Universidad Nacional Autónoma de México (UNAM), A.P. 70-264, 04510 México, D.F., Mexico

${ }^{4}$ Sydney Institute for Astronomy, School of Physics, The University of Sydney, NSW 2006, Australia

${ }^{5}$ Dunlap Institute for Astronomy and Astrophysics, University of Toronto, Toronto, ON M5S 3H4, Canada defined as the gradient of the polarization angle $\chi$ against $\lambda^{2}$

$$
\mathrm{RM}=\frac{\Delta \chi}{\Delta \lambda^{2}} .
$$

Assuming a simple case in which all radiation undergoes the same amount of rotation RM corresponds to the Faraday Depth $\phi$ computed as (Burn (1966))

$$
\phi\left(z_{s}\right)=8.1 \times 10^{5} \int_{z_{s}}^{0} \frac{n_{e}(z) B_{\|}(z)}{(1+z)^{2}} \frac{d l}{d z} d z
$$

where $\phi$ is in units of $\mathrm{rad} / \mathrm{m}^{2}$, the free electron number density $n_{e}$ in units of $\mathrm{cm}^{-3}$, the magnetic field component along the line of sight $B_{\|}$in units of $\mathrm{G}$ and the comoving path increment per unit redshift $d l / d z$ in units of pc. Thus RM can be used to estimate the parallel component of the magnetic field along the observed line of sight.

Unfortunately, the measurement of the RM gives no information about where along the line of sight the Faraday Rotation is occurring, i.e. intrinsically to the radio source or its immediate surroundings, during passage through an intervening system along the line of sight, or locally within our own Galaxy. If different parts of a source suffer different amounts of Faraday Rotation, e.g. if there is a spread in $\phi$ caused by passing through an inhomogeneous foreground screen, then because the polarization is a polar quantity, the net effect may be to reduce the overall polarization of the source (Burn (1966), see also Gardner \& Whiteoak (1966), Sokoloff et al. (1998)). These depolarization effects can cause non-linearities in the slope of $\chi$ against $\lambda^{2}$ and make the RM poorly defined. 
Kronberg et al. (2008) presented evidence that the distribution of the apparent RM of a large sample of radio quasars became broader with redshift. One possibility was that this was due to the increased probability, as the redshift increases, that a given line of sight intersects intervening material. To test this hypothesis, Bernet et al. (2008) obtained high resolution optical spectra of a set of 76 quasars for which RM were available. That analysis showed that, indeed, systems with strong MgII absorption in their spectra had a broader distribution of RM than those without, and argued that this was not being caused by a secondary correlation of the presence of MgII with e.g. redshift. Bernet et al. (2008) estimated that the lines of sight with strong MgII absorption were suffering, statistically, an increased $|\mathrm{RM}| \approx 40 \mathrm{rad} / \mathrm{m}^{2}$. Applying a crude estimate for the free electron column density led to an estimate of magnetic field strengths of order $10 \mu G$ in the absorption systems, which are typically at redshift $z \approx 1$.

Furthermore by taking optical images of those quasars with MgII absorption, the absorbing systems could be associated with individual galaxies (Bernet et al. (2013)). High values of RM were found only for MgII systems that lay within 50 projected kpc. These findings were very interesting in the context of the results of Bordoloi et al. (2011) who had mapped MgII absorption around galaxies at similar redshifts and shown that most MgII absorption at these redshifts occurs in biconical bipolar regions extending out to $50 \mathrm{kpc}$ around star forming galaxies. Taken together they suggested that the observed magnetic fields were being transported out of the galaxies by winds. This would have implications for not only the development of magnetic fields in galaxies but also for the origin of magnetic fields in the circumgalactic and even intergalactic media (Bhat \& Subramanian (2013), Shukurov et al. (2006)).

Meanwhile new large catalogs of RM measurements became available, especially from $1.4 \mathrm{GHz}$ surveys (e.g. Taylor et al. (2009), see also Farnes et al. (2014)). Bernet et al. (2012) compared the new RM measurements from Taylor et al. (2009), which overlapped partly with the sample they have used (Kronberg et al. (2008)), and found that there were significant differences. Furthermore, with the new RM data the excess RM associated with lines of sight with MgII absorption could not be recovered.

The new RM samples were however measured at a relatively low frequency of $1.4 \mathrm{GHz}$, while the previous RM measurements were at higher frequencies of $5 \mathrm{GHz}$. Bernet et al. (2012) showed, using a simple toy model that the presence of inhomogeneous screens could lead to substantial depolarization towards lower frequencies which could introduce complex behavior into the wavelengthdependence of the polarization angle and thus mask the intervener effect when RM measurements from low frequency surveys were used. This was later tested in Bernet et al. (2013) who claimed that depolarization was strongly associated with impact parameter for the MgII systems.

Later Joshi \& Chand (2013) also used the RM catalog of Hammond et al. (2012), which is a crossmatch of the Taylor et al. (2009) catalog with several QSO redshift catalogs and found a marginal excess of RM associated with MgII absorption. Also, Farnes et al. (2015) con- firmed, with a much larger sample of around 600 objects which they had compiled from their catalog (Farnes et al. (2014)), the association between excess RM and the presence of MgII absorption along the line of sight. They also found that the association was only present if only flat spectrum sources were considered. This could be because they will be smaller in size and therefore have their radio and optical sight lines more closely aligned. Bernet et al. (2008) did not have this problem since their sample had been selected to be both compact and to have only small offsets between radio and optical emission.

With the advent of new technology it has become possible to further improve the quality of the radio data by measuring polarization at high spectral resolution over a long baseline in wavelength. In particular, such data allows Faraday Rotation Measure Synthesis (RM Synthesis). RM Synthesis transforms the complex representation of the polarization structure

$$
\mathbf{P}\left(\lambda^{2}\right)=P\left(\lambda^{2}\right) e^{2 i \chi\left(\lambda^{2}\right)}=Q\left(\lambda^{2}\right)+i U\left(\lambda^{2}\right)
$$

into the complex Faraday Depth distribution

$$
\mathbf{F}(\phi)=\frac{1}{\pi} \int_{-\infty}^{\infty} \mathbf{P}\left(\lambda^{2}\right) e^{-2 i \phi \lambda^{2}} d \lambda^{2}
$$

where $P$ is the polarized flux density, $\chi$ the polarization angle and $Q$ and $U$ the corresponding stokes parameters. $\mathbf{F}(\phi)$ is again a complex distribution and can be written as

$$
\mathbf{F}(\phi)=F(\phi) e^{2 i \psi(\phi)}=\tilde{Q}(\phi)+i \tilde{U}(\phi)
$$

where the amplitude $F(\phi)$ will be referred to as the Faraday Depth (FD) distribution and $\psi(\phi)$ as the "initial phase" distribution. $F(\phi)$ is also called Faraday Dispersion Function or Faraday Spectrum in different literatures. $\tilde{Q}$ and $\tilde{U}$ are completely analogous to $Q$ and $U$ but in $\phi$ space.

The FD distribution $F(\phi)$ quantifies how much linearly polarized flux density has been subject to a certain Faraday Depth $\phi$. The initial phase distribution $\psi(\phi)$ represents the effective angle of polarization (before any Faraday rotation takes place) of the linearly polarized emission that lies at a given Faraday Depth $\phi$. By effective angle, we mean the angle of the polar sum of the linearly polarized components.

A given source may exhibit a range of $\phi$ because one or, most likely, both of the following conditions hold. There must be either (a) different $\phi$ across the source (i.e in the plane of the sky) within the telescope angular resolution due to variations across the emitting source or across an intervening screen or (b) a contribution to $\phi$ along the line of sight within the emitting source.

RM Synthesis is a useful tool for studying Faraday Rotation effects, and depolarization effects, for radio sources. However, RM Synthesis requires dense measurements over a continuous frequency band to get a reasonable coverage of $\mathbf{P}\left(\lambda^{2}\right)$ (see Beck et al. (2012) for a summary of the Faraday Depth resolution of current and future radio telescopes). This has not been available in earlier works dealing with intermediate redshift magnetic fields. The goal of the current paper has been to obtain the FD distribution $F(\phi)$ of a substantial fraction of the radio sources for which we have high quality information on MgII absorption and which we used in earlier papers 
Table 1

Observed sources

\begin{tabular}{|c|c|c|c|c|c|c|c|}
\hline Name & RA (J2000) & Dec. (J2000) & $z_{\mathrm{QSO}}$ & MgII Abs. & $z_{\mathrm{Abs}}$ & Instrument & Obs. block ${ }^{1}$ \\
\hline PKS0130-17 & 01:32:43.4 & $-16: 54: 48$ & 1.02 & yes & 0.51 & ATCA & A-SB1 \\
\hline PKS0135-247 & $01: 37: 38.3$ & $-24: 30: 54$ & 0.84 & yes & 0.47 & ATCA & A-SB1 \\
\hline PKS0139-09 & $01: 41: 25.8$ & $-09: 28: 44$ & 0.73 & yes & 0.50 & ATCA & A-SB1 \\
\hline $3 \mathrm{C} 057$ & 02:01:57.2 & $-11: 32: 33$ & 0.67 & no & & ATCA & A-SB1 \\
\hline PKS0202-17 & 02:04:57.7 & $-17: 01: 19$ & 1.74 & yes & 0.52 & ATCA & A-SB1 \\
\hline PKS0332-403 & $03: 34: 13.7$ & $-40: 08: 25$ & 1.45 & yes & 1.21 & ATCA & A-SB3 \\
\hline PKS0402-362 & 04:03:53.7 & $-36: 05: 02$ & 1.42 & yes & 0.80 & ATCA & A-SB2 \\
\hline PKS0422-380 & $04: 24: 42.4$ & $-37: 56: 22$ & 0.78 & no & & ATCA & A-SB3 \\
\hline PKS0426-380 & $04: 28: 40.4$ & $-37: 56: 20$ & 1.11 & yes & 0.56 & ATCA & A-SB2 \\
\hline PKS0506-61 & 05:06:43.9 & $-61: 09: 41$ & 1.09 & yes & 0.92 & ATCA & A-SB3 \\
\hline PKS0839+18 & 08:42:05.1 & $+18: 35: 42$ & 1.27 & yes & 0.71 & VLA & V-SB1 \\
\hline $4 \mathrm{C}+01.24$ & 09:09:10.1 & $+01: 21: 36$ & 1.02 & yes & 0.54 & VLA & V-SB1 \\
\hline $4 \mathrm{C}+02.27$ & $09: 35: 18.2$ & $+02: 04: 16$ & 0.65 & no & & VLA & V-SB1 \\
\hline $\mathrm{OK}+186$ & $09: 54: 56.8$ & $+17: 43: 32$ & 1.48 & no & & VLA & V-SB1 \\
\hline $4 C+19.34$ & $10: 24: 44.8$ & $+19: 12: 21$ & 0.83 & yes & 0.53 & VLA & V-SB1 \\
\hline $4 \mathrm{C}+06.41$ & $10: 41: 17.2$ & $+06: 10: 17$ & 1.27 & yes & 0.44 & VLA & V-SB1 \\
\hline $3 \mathrm{C} 245$ & $10: 42: 44.5$ & $+12: 03: 32$ & 1.03 & yes & 0.66 & VLA & V-SB1 \\
\hline $4 C+20.24$ & $10: 58: 17.9$ & $+19: 51: 51$ & 1.11 & yes & 0.86 & VLA & V-SB1 \\
\hline PKS1111+149 & $11: 13: 58.7$ & $+14: 42: 27$ & 0.87 & yes & 0.65 & VLA & V-SB1 \\
\hline PKS1127-14 & 11:30:07.1 & $-14: 49: 27$ & 1.19 & no & & ATCA & A-SB2 \\
\hline PKS1143-245 & $11: 46: 08.1$ & $-24: 47: 32$ & 1.94 & yes & 1.25 & ATCA & A-SB3 \\
\hline PKS1157+014 & $11: 59: 44.8$ & $+01: 12: 07$ & 1.99 & yes & 1.94 & VLA & V-SB1 \\
\hline $4 \mathrm{C}+13.46$ & $12: 13: 32.1$ & $+13: 07: 20$ & 1.14 & yes & 0.77 & VLA & V-SB1 \\
\hline $4 \mathrm{C}-02.55$ & 12:32:00.0 & $-02: 24: 05$ & 1.05 & yes & 0.40 & VLA & V-SB1 \\
\hline PKS1244-255 & $12: 46: 46.9$ & $-25: 47: 48$ & 0.63 & yes & 0.49 & ATCA & A-SB2 \\
\hline $\mathrm{ON}+187$ & $12: 54: 38.3$ & $+11: 41: 06$ & 0.87 & no & & VLA & V-SB3 \\
\hline $4 \mathrm{C}-00.50$ & $13: 19: 38.7$ & $-00: 49: 41$ & 0.89 & no & & VLA & V-SB3 \\
\hline $4 \mathrm{C}+19.44$ & $13: 57: 04.4$ & $+19: 19: 08$ & 0.72 & yes & 0.46 & VLA & V-SB3 \\
\hline $3 \mathrm{C} 298$ & $14: 19: 08.2$ & $+06: 28: 35$ & 1.44 & no & & VLA & V-SB3 \\
\hline PKSB1419-272 & $14: 22: 49.0$ & $-27: 27: 56$ & 0.99 & yes & 0.56 & VLA & V-SB3 \\
\hline OQ+135 & $14: 23: 30.1$ & $+11: 59: 51$ & 1.61 & yes & 1.36 & VLA & V-SB3 \\
\hline $4 \mathrm{C}-05.62$ & $14: 56: 41.5$ & $-06: 17: 42$ & 1.25 & no & & VLA & V-SB3 \\
\hline 4C-05.64 & $15: 10: 53.6$ & $-05: 43: 07$ & 1.19 & yes & 0.38 & VLA & V-SB3 \\
\hline $4 \mathrm{C}+05.64$ & $15: 50: 35.3$ & $+05: 27: 11$ & 1.42 & no & & VLA & V-SB3 \\
\hline PKS1615+029 & $16: 17: 49.9$ & $+02: 46: 44$ & 1.34 & yes & 0.53 & VLA & V-SB3 \\
\hline OW-174 & $20: 47: 19.7$ & $-16: 39: 06$ & 1.93 & yes & 1.33 & VLA & V-SB2 \\
\hline OX-325 & $21: 18: 10.7$ & $-30: 19: 15$ & 0.98 & no & & VLA & V-SB2 \\
\hline PKS2134+004 & $21: 36: 38.6$ & $+00: 41: 55$ & 1.94 & yes & 0.63 & VLA & V-SB2 \\
\hline OX-173 & $21: 46: 23.0$ & $-15: 25: 44$ & 0.70 & no & & ATCA & A-SB1 \\
\hline $4 C+6.69$ & $21: 48: 05.4$ & $+06: 57: 39$ & 0.99 & yes & 0.79 & VLA & V-SB2 \\
\hline OX-192 & 21:58:06.3 & $-15: 01: 09$ & 0.67 & yes & 0.39 & ATCA & A-SB1 \\
\hline PKS2204-54 & $22: 07: 43.7$ & $-53: 46: 34$ & 1.21 & yes & 0.69 & ATCA & A-SB1 \\
\hline $4 C-3.79$ & $22: 18: 52.0$ & $-03: 35: 37$ & 0.90 & no & & VLA & V-SB2 \\
\hline PKS2223-05 & $22: 25: 47.3$ & $-04: 57: 02$ & 1.40 & yes & 0.85 & VLA & V-SB2 \\
\hline PKS2227-08 & $22: 29: 40.0$ & $-08: 32: 54$ & 1.56 & no & & ATCA & A-SB1 \\
\hline $4 \mathrm{C}+11.69$ & $22: 32: 36.4$ & $+11: 43: 50$ & 1.04 & yes & 0.74 & VLA & V-SB2 \\
\hline PKS2243-123 & $22: 46: 18.2$ & $-12: 06: 52$ & 0.63 & no & & ATCA & A-SB1 \\
\hline $3 \mathrm{C} 454.3$ & $22: 53: 57.7$ & $+16: 08: 53$ & 0.86 & no & & VLA & V-SB2 \\
\hline PKS2326-477 & $23: 29: 17.7$ & $-47: 30: 19$ & 1.30 & yes & 0.43 & ATCA & A-SB1 \\
\hline
\end{tabular}

${ }^{1}$ See text for specifications.

(Bernet et al. (2008), Bernet et al. (2010), Bernet et al. (2013)).

This paper is structured as follows. In Section 2, we first describe our sample as well as the new observations, data reduction and measurements. We also describe how the RM Synthesis has been carried out and provide a brief review of the general interpretation of this relatively unfamiliar type of data. In Section 3 we introduce some parameters which quantify structures in the polarization and in the FD distribution and which we will use in the later parts of the paper. In Section 4 we fist look at whether these are correlated with Galactic latitude which would indicate a potential Galactic origin. We then turn in Section 5 to examine the association with intervening MgII absorption and begin the development of the main results of the paper. We first look at the analogues of the previous analyses in Bernet et al. (2008) regarding the distribution of overall RM and depolarization with the presence of MgII absorption. We find a strong association of intervening MgII with various depolarization signatures. Turning to examine the structure within the FD distribution $F(\phi)$, which we would expect to cause the depolarization, we find surprisingly little correlation between this structure and MgII. This leads us to explore the links between $F(\phi)$ and different manifestations of depolarization, and enables us to identify those features in $F(\phi)$ which arise in intervening systems, and those which are likely to be associated with the intrinsic properties of the sources. Finally in Section 6 we discuss the implications which arise from our results and in Section 7 we summarize our findings. 


\section{DATA}

\subsection{Sample}

The sample of quasars is selected from Bernet et al. (2008) and therefore has exactly the same MgII information available for every quasar from VLT UVES spectroscopy. Some sources from Bernet et al. (2008) were not observed simply because of scheduling constraints at the telescopes. With few exceptions, quasars with Dec $>10^{\circ}$ were observed with the Karl G. Jansky Very Large Array (VLA) while those south of this were observed with the Australia Telescope Compact Array (ATCA). 34 objects were observed with VLA and 25 with ATCA. However, 4 objects observed with VLA and 6 objects observed with ATCA are not considered further for this analysis. The 4 VLA objects which are thrown out are 3C208, 3C281, 4C-06.35 and PKS1424-11. For those objects it turned out that the core is too faint (and thus too low in $\mathrm{S} / \mathrm{N}$ ) for our analysis. We believe that in prior observations the radio lobes have been confused with the core. 6 ATCA objects were discarded because they are sufficiently resolved such that a phase calibration has not been possible. The decision to discard those objects was taken without any knowledge about their MgII absorption properties to avoid any bias in the results.

A list of the final set of 49 sources is provided in Table 1 including their position, their redshift, the redshift of their absorbers if present, and a log of observations. We declare an MgII absorbing system to be present if an absorption line with rest frame equivalent width $W_{0} \geq$ $0.1 \AA$ is detected along the line of sight. This deviates slightly from the earlier analysis in Bernet et al. (2008) in which a cut at $W_{0}=0.3 \AA$ has been applied. We will discuss our choice later in this paper. Consequently the sample with interveners contains 33 objects while the sample with clean lines of sight contains 16 objects. Similar results are found if attention is restricted only to stronger sources, albeit at reduced significance.

\subsection{VLA Observations, data reduction and flux density measurements}

The objects were observed in three scheduling blocks (V-SB, cf. Table 1) in configuration A with a maximum baseline of $36.4 \mathrm{~km}$. V-SB1 was taken on 2014 March 4, V-SB2 on 2014 April 24 and V-SB3 on 2014 April 23 (Proposal ID: VLA/14A-144, PI: F. Miniati). The observations were taken using the 8 bit sampler in L, S and $\mathrm{C}$ band, respectively, and cover with full polarization the frequency range between approximately 1 and $6 \mathrm{GHz}$, implying a maximum frequency resolution of $2 \mathrm{MHz}$ in $\mathrm{L}$ band and $1 \mathrm{MHz}$ in $\mathrm{S}$ and $\mathrm{C}$ band.

CASA version 4.4.0 (McMullin et al. (2007)) has been used for all calibration steps. 3C286 served as bandpass and flux calibrator in all three scheduling blocks. Since the objects are distributed over the sky individual phase calibrators had to be chosen for each object. For nearby objects the same calibrator has been used and bright and unresolved objects have been calibrated by themselves. The chosen phase calibrators are listed in Table 2. Sometimes sources are resolved in $\mathrm{L}$ and $\mathrm{S}$ band but not in $\mathrm{C}$ band. In those cases phase calibrators have been used only for $\mathrm{L}$ and $\mathrm{S}$ band. The phase calibrators were always observed both, before and after the observation of the target. 3C286 also serves as the polarization angle
Table 2

Phase Calibrators for the VLA sources

\begin{tabular}{|c|c|}
\hline Name & Phase Calibrators \\
\hline $\mathrm{PKS} 0839+18$ & self \\
\hline $4 \mathrm{C}+01.24$ & self \\
\hline $4 \mathrm{C}+02.27$ & $4 \mathrm{C}+01.24$ \\
\hline $\mathrm{OK}+186$ & self \\
\hline $4 \mathrm{C}+19.34$ & $\mathrm{OK}+186$ \\
\hline $4 \mathrm{C}+06.41$ & self \\
\hline $3 \mathrm{C} 245$ & $\mathrm{~J} 1120+1420$ \\
\hline $4 \mathrm{C}+20.24$ & $\mathrm{~J} 1120+1420$ \\
\hline PKS1111+149 & $\mathrm{J} 1120+1420$ \\
\hline PKS1157+014 & $\mathrm{J} 1224+0330$ \\
\hline $4 \mathrm{C}+13.46$ & $\mathrm{~J} 1224+0330$ \\
\hline $4 \mathrm{C}-02.55$ & $\mathrm{~J} 1224+0330$ \\
\hline $\mathrm{ON}+187$ & self \\
\hline $4 \mathrm{C}-00.50$ & J1354-0206 \\
\hline $4 \mathrm{C}+19.44$ & self \\
\hline $3 \mathrm{C} 298$ & $\mathrm{~J} 1415+1320$ \\
\hline PKSB1419-272 & J1438-2204 \\
\hline $\mathrm{OQ}+135$ & $4 \mathrm{C}+19.44$ \\
\hline $4 \mathrm{C}-05.62$ & J1513-1012 \\
\hline $4 \mathrm{C}-05.64$ & J1513-1012 \\
\hline $4 C+05.64$ & PKS1615+029 \\
\hline PKS1615+029 & self \\
\hline OW-174 & J2110-1020/self ${ }^{1}$ \\
\hline OX-325 & J2138-2439 \\
\hline PKS2134+004 & self \\
\hline $4 \mathrm{C}+6.69$ & self \\
\hline $4 \mathrm{C}-3.79$ & $\mathrm{~J} 2212+0152 /$ self $^{1}$ \\
\hline PKS2223-05 & $\mathrm{J} 2212+0152 / 4 \mathrm{C}-3.79^{1}$ \\
\hline $4 \mathrm{C}+11.69$ & $\mathrm{~J} 2250+1419 /$ self $^{1}$ \\
\hline $3 \mathrm{C} 454.3$ & $\mathrm{~J} 2250+1419 /$ self $^{1}$ \\
\hline
\end{tabular}

${ }^{1}$ First calibrator used for $\mathrm{L}$ and $\mathrm{S}$ band and second calibrator used for $\mathrm{C}$ band.

calibrator (Perley \& Butler (2013)). The unpolarized source J1407+2827 was used to correct for polarization leakage. The residual instrumental polarization is safely below $0.3 \%$.

Data that was obviously affected by radio frequency interference (RFI) have been flagged by hand. After recalibration further flagging has been applied by running the flagdata command in rflag mode (Greisen (2003)). Altogether around $30 \%$ of the data have been flagged for each source, mostly in the $\mathrm{L}$ band.

Since only the quasar itself is interesting for this work we synthesize images just in a small window of $30^{\prime \prime} \times 30^{\prime \prime}$ around it. The images have been cleaned according to the Cotton-Schwab algorithm (Schwab (1984), Clark (1980), Högbom (1974)) and applying Briggs weighting with robust parameter $R=0$. To ensure that we do not get any flux leakage from other sources situated outside the synthesized window it has been checked, for each source, whether there are other bright sources within the primary beam and it has never been the case. In a few cases where the source is extended the image sizes have been matched appropriately. In L band images are made in steps of around $16 \mathrm{MHz}$ and in $\mathrm{S}$ and $\mathrm{C}$ Band in steps of $128 \mathrm{MHz}$. Self calibration has been applied in all frames.

To make the images at different frequencies comparable, all images have been smoothed to the beam size of the lowest resolution image, typically around $1.3^{\prime \prime}$. To retrieve the flux density the brightness has been integrated over an aperture covering the FWHM of the synthesized beam centered around the maximum flux density pixel in 


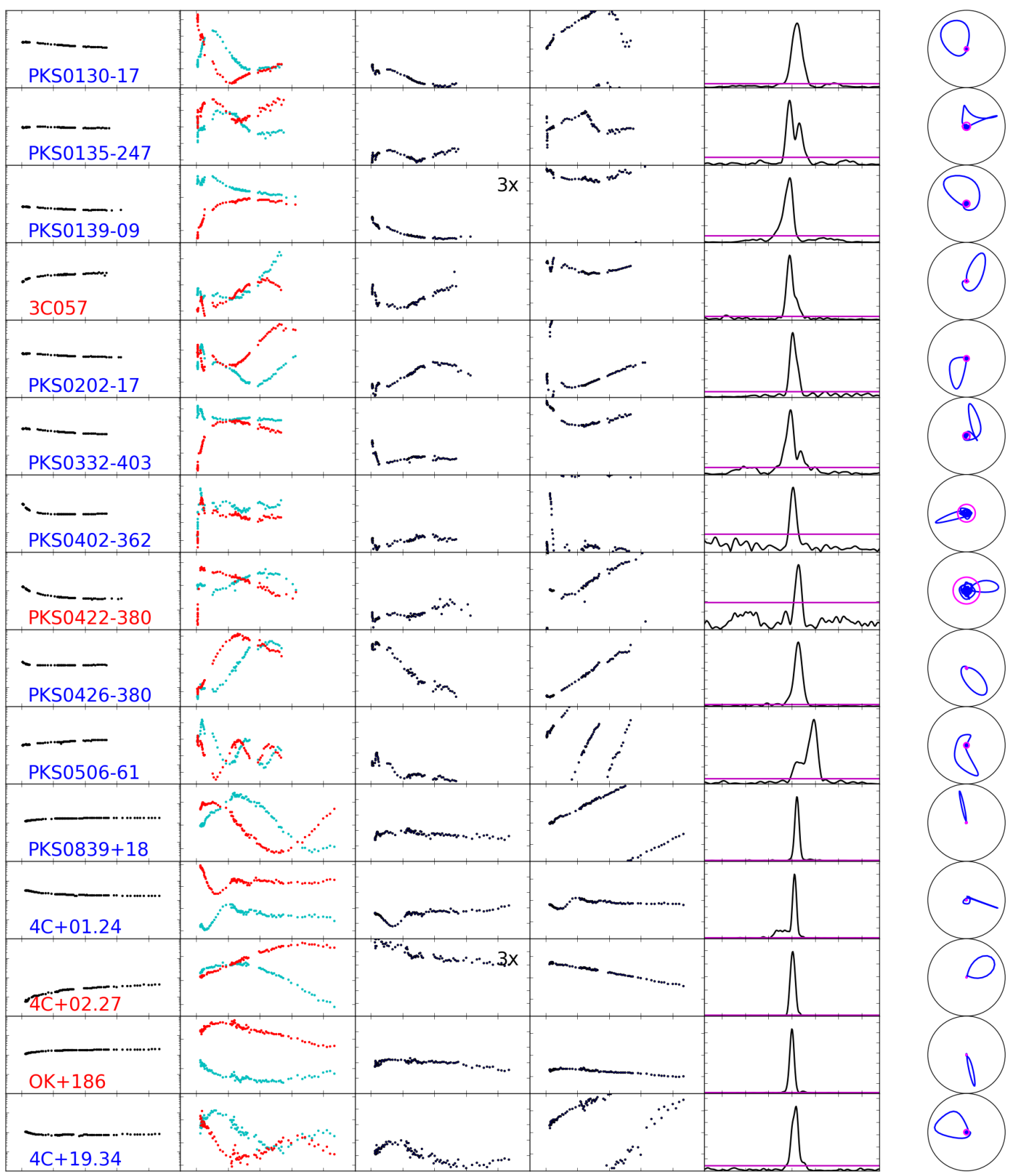

Figure 1. Measured and derived data points. Each row represents one object specified in Column (i). Objects with intervener are indicated by blue and those without by red font color. The columns represent from left to right: (i) $I\left(\lambda^{2}\right)[0.1 \mathrm{Jy}, 100 \mathrm{Jy}]$, (ii) $U\left(\lambda^{2}\right)$ in cyan and $Q\left(\lambda^{2}\right)$ in red [arbitrary], (iii) $\Pi\left(\lambda^{2}\right)[0,10 \%]$, (iv) $\chi\left(\lambda^{2}\right)\left[-90^{\circ}, 90^{\circ}\right]$, (v) $F(\phi)$ [arbitrary], (vi) $\mathbf{F}(\phi)$. Square brackets indicate the range of the $\mathrm{y}$-axes. Column (i) is scaled logarithmically and the range of Column (iii) is enlarged to [0, 30\%] when indicated by " $3 \mathrm{x}$ " on the top right corner of the panel. The range of x-axes are $\left[0,0.1 \mathrm{~m}^{2}\right]$ for Columns (i)-(iv) and $\left[-750 \mathrm{rad} / \mathrm{m}^{2}, 750 \mathrm{rad} / \mathrm{m}^{2}\right]$ for Column (v). The $F(\phi)$ distributions in Column (v) are shifted by the Oppermann et al. (2015) estimates of the Galactic contribution to $\phi$. The magenta lines in Column (v) and (vi) represent the $5 \sigma$ noise level obtained by the sigma clipping algorithm. To make use of the whole circle $\chi(\phi)$, which is defined between $-\pi$ and $\pi$, is multiplied by 2 in Column (vi). 


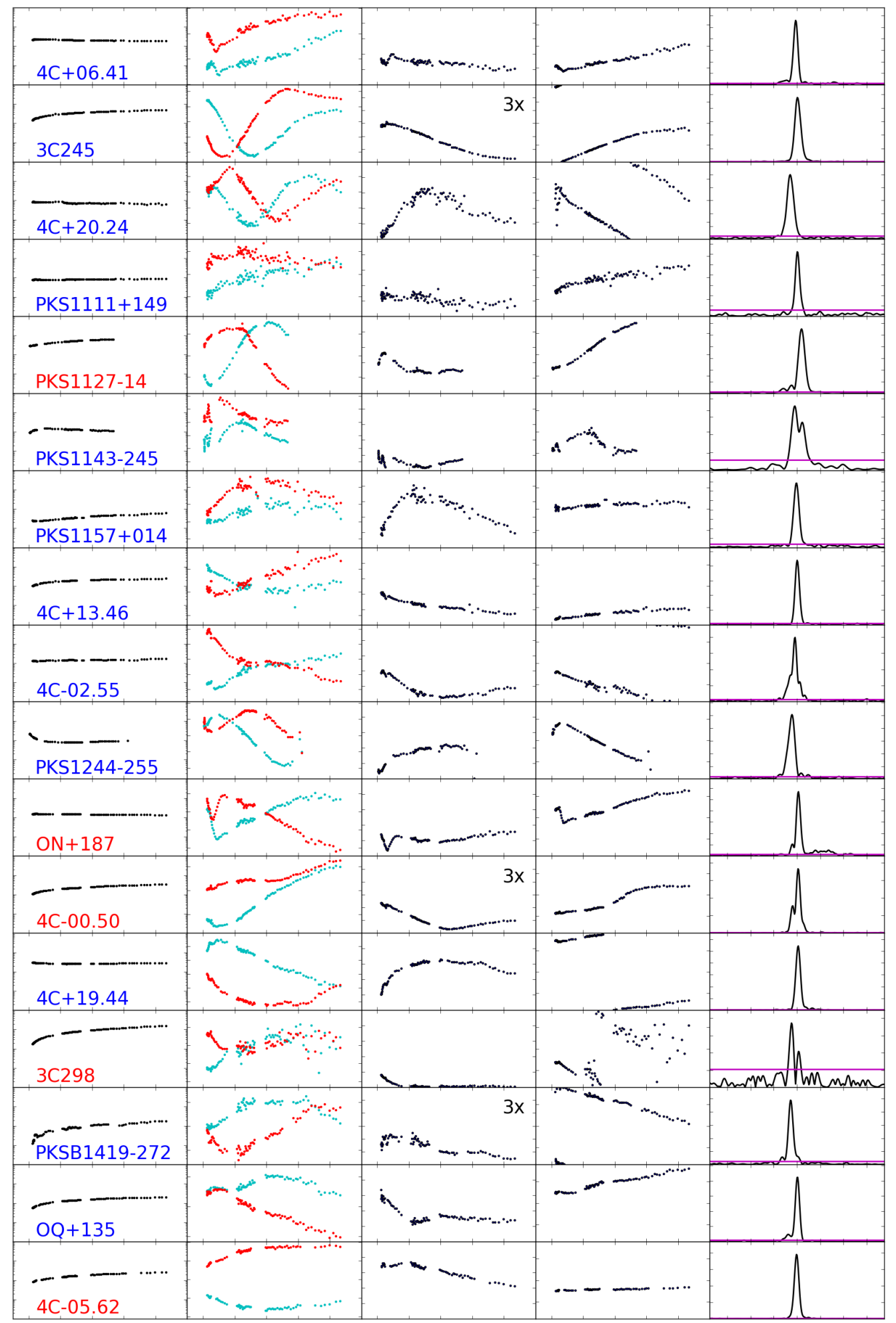

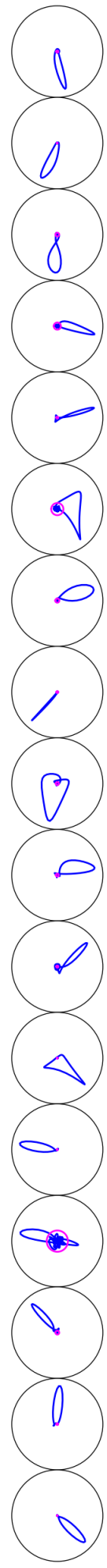

Figure 2. Compare to Figure 1. 


\begin{tabular}{|c|c|c|c|c|}
\hline $\begin{array}{l}-\ldots \ldots \\
4 C-05.64\end{array}$ & $r$ & $=$ & $1-4_{m=1}+\ldots . . . . . . .$. & 1 \\
\hline 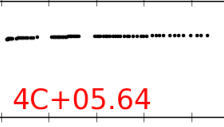 & $y$ & $-\infty,-\infty$ & & \\
\hline 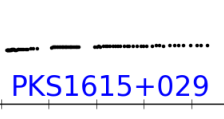 & & $n_{i=1}-\ldots$ & & \\
\hline oW-174 & 3 & $-\infty$ & & \\
\hline OX-325 & & 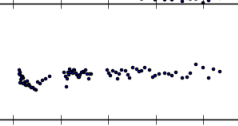 & & 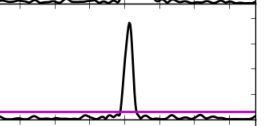 \\
\hline PKS2134+004 & & & & $\sqrt{1}$ \\
\hline ox-173. & & 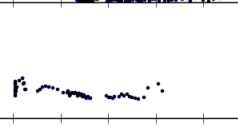 & & 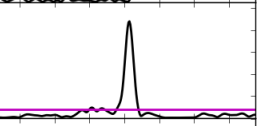 \\
\hline$---\ldots$ & 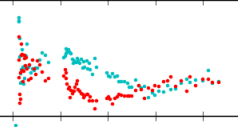 & & $\vdots$ & Ithmon \\
\hline Ox-192 & & $p^{2}$ & & \\
\hline PKS2204-54 & & & & \\
\hline $\begin{array}{l}-- \\
4 C-3.79\end{array}$ & 6 & 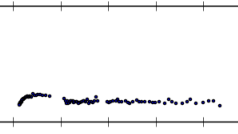 & $m$ & \\
\hline PKS2223-05 & & & & \\
\hline PKS2227-08 & is & $i^{2}-m$ & & \\
\hline $4 C+11.69$ & & & & \\
\hline PKS2243-123 & $m-m$ & 1 & $r$ & 1 \\
\hline $3 C 454.3$ & & -2 & & \\
\hline PKS2326-477 & $r$ & $r^{n-m}$ & $\ldots$ & \\
\hline
\end{tabular}
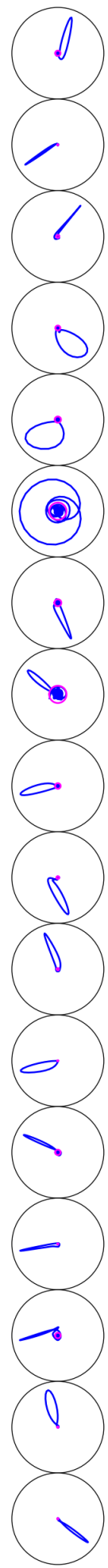

Figure 3. Compare to Figure 1. 
the $I$ frame. Subsequently $Q$ and $U$ flux densities have been obtained by integrating over the same aperture in $Q$ and $U$. The obtained flux densities have been converted to total flux densities assuming a two dimensional Gaussian profile of the sources. The retrieved data ( $I$, $Q$ and $U$ parameter) as well as the derived polarization fraction $\Pi$ and polarization angle $\chi$ are shown in the Columns (i)-(iv) of Figures 1-3.

\subsection{ATCA observations, data reduction and flux density measurements}

The ATCA observations took place on 2014 May 3 (ASB1, cf. Table 1) and 2013 May 14 (A-SB2), 15 (ASB3) in configuration $6 \mathrm{C}$ corresponding to a maximum baseline of $6 \mathrm{~km}$. The observed frequency range was 1.1 - $3.1 \mathrm{GHz}$ (16 cm band), 4.0 - $6.0 \mathrm{GHz}$ (4 cm band) and $8.8-10 \mathrm{GHz}$ (4 cm band) with a resolution of $1 \mathrm{MHz}$ (Proposal ID: C2769, PI: M. L. Bernet). Each source has been observed with at least three cuts in the uv-plane.

The reduction package MIRIAD (Sault et al. (1995)) has been used to carry out the calibration. For sources in A-SB1 PKSB1934-638 served as the bandpass calibrator in the $16 \mathrm{~cm}$ band and PKSB1921-293 in the $4 \mathrm{~cm}$ band while for sources in A-SB2 and A-SB3 PKS0823-500 served as the bandpass calibrator. Furthermore PKSB1934-638 is used as the flux calibrator for all sources. We observed repeatedly PKSB2326-477 and PKSB2005-489 during A-SB1 and PKS1903-802 during A-SB2 and A-SB3 for phase and polarization leakage calibration. However, since the phase calibration solutions turned out to be unsatisfying, we relied solely on self calibration for all sources. This is the reason why some sources were discarded. The pgflag command has been used to auto-flag corrupted data. Some manual flagging has been applied afterwards.

$I, Q$ and $U$ Images of $2^{\prime} \times 2^{\prime}$ frames around the objects are synthesized in steps of $16 \mathrm{MHz}$ over the whole observed frequency band applying natural weighting. Cleaning has been carried out with the clean command in any mode. All frames have been smoothed to the same resolution, typically around $10^{\prime \prime}$, and it has been ensured that they contain only one single unresolved source. The flux density of that source has been determined by measuring the maximum brightness of the frames. The data points of the $4 \mathrm{~cm}$ band images have been binned in steps of $128 \mathrm{MHz}$ to have similar data spacings as the VLA objects.

The retrieved data $(I, Q$ and $U)$ as well as the derived polarization fraction $\Pi$ and polarization angle $\chi$ are shown in the Columns (i)-(iv) of Figures 1-3. We conclude that the structure of $I, Q, U$ is comparable between sources observed at VLA with sources observed at ATCA ensuring the consistency of our sample.

\subsection{Rotation Measure Synthesis}

RM Synthesis was carried out using the code provided by Brentjens \& de Bruyn (2005). The RM Synthesis is calculated with uniform weighting and relative to $\lambda^{2}=0$. Subsequently, the RM-CLEAN algorithm has been applied, following Heald et al. (2009). The effects of RM-CLEAN are shown in Figure 4 for four objects. PKS0839+18 and PKS2326-477 are sources with simple FD distributions that were observed with the VLA and
ATCA, respectively. 4C+01.24 and PKS0135-247 represent sources with more complex FD distributions from the VLA and ATCA, respectively. The second row shows the dirty $F(\phi)$, that is before RM-CLEAN is applied, and the third row shows the clean $F(\phi)$ after the application of RM-CLEAN.

The first row shows the Rotation Measure Spread Functions (RMSF) of the objects. The RMSF is the function with which the true FD distribution is convolved due to the finite sampling of the data in $\lambda^{2}$ space. After running RM-CLEAN the RMSF corresponds to a Gaussian whereby its FWHM can be estimated as (Brentjens \& de Bruyn (2005))

$$
\delta \phi \approx \frac{2 \sqrt{3}}{\Delta \lambda^{2}}
$$

where $\Delta \lambda^{2}$ is the observed frequency bandwidth in $\lambda^{2}$ space. For ease of comparison later on we introduce

$$
\sigma_{\mathrm{RMSF}}=\frac{\delta \phi}{2 \sqrt{2 \ln 2}}
$$

defined as the standard deviation of the clean Gaussian RMSF. $\sigma_{\mathrm{RMSF}}$ corresponds to the "resolution" of the data in $\phi$ space. The $\sigma_{\mathrm{RMSF}}$ for the VLA data is around $17 \mathrm{rad} / \mathrm{m}^{2}$ and for the ATCA data it is around $24 \mathrm{rad} / \mathrm{m}^{2}$.

Despite some known shortcomings of the RM-CLEAN method to accurately reconstruct complex FD distributions (Sun et al. (2015)) we dispense with more sophisticated but complicated methods (e.g. Frick et al. (2010), Li et al. (2011), Farnsworth et al. (2011), O'Sullivan et al. (2012), Schnitzeler \& Lee (2015)). We regard the method adopted to be sufficient for our purposes since our analysis aims primarily to separate sources with simple FD distributions from those with complex ones. As will become clear later in the paper, our conclusions do not depend on small components in the FD distribution.

Historically it has been conventional to use the Stokes parameter $Q$ and $U$ as input for the RM Synthesis, according to the definition in Equation 4. Recently, however, the fractional parameter $q=Q / I$ and $u=U / I$ has been utilized (Anderson et al. (2015)). There are good reasons to do that, especially when one is dealing with steep spectral index sources. Since our sources, however, mostly have flat spectra, we do not expect the differences of the two methods to be severe. Indeed we have tried out both methods and it turns out that the conclusions we draw in this paper hold whatever method we use. For the presentation of the results in this paper we choose to stick with the conventional method, i.e. utilizing $Q$ and $U$, and mention the results with $q$ and $u$.

The obtained FD distributions $F(\phi)$ are shown in the panels of Column (v) of Figure 1-3 for all 49 sources. Although the FD distributions are synthesized between $-1500 \mathrm{rad} / \mathrm{m}^{2}$ and $1500 \mathrm{rad} / \mathrm{m}^{2}$ we show only the range between $-750 \mathrm{rad} / \mathrm{m}^{2}$ and $750 \mathrm{rad} / \mathrm{m}^{2}$ since we do not detect any significant signal outside of this window. We adopt the Galactic RM contribution estimates from Oppermann et al. (2015) and shift the overall FD distributions accordingly.

The polar plots in the panels of Column (vi) represent the complex FD distribution $\mathbf{F}(\phi)$. In this, the azimuthal angle represents the initial phase $\psi$, i.e. the polarization 


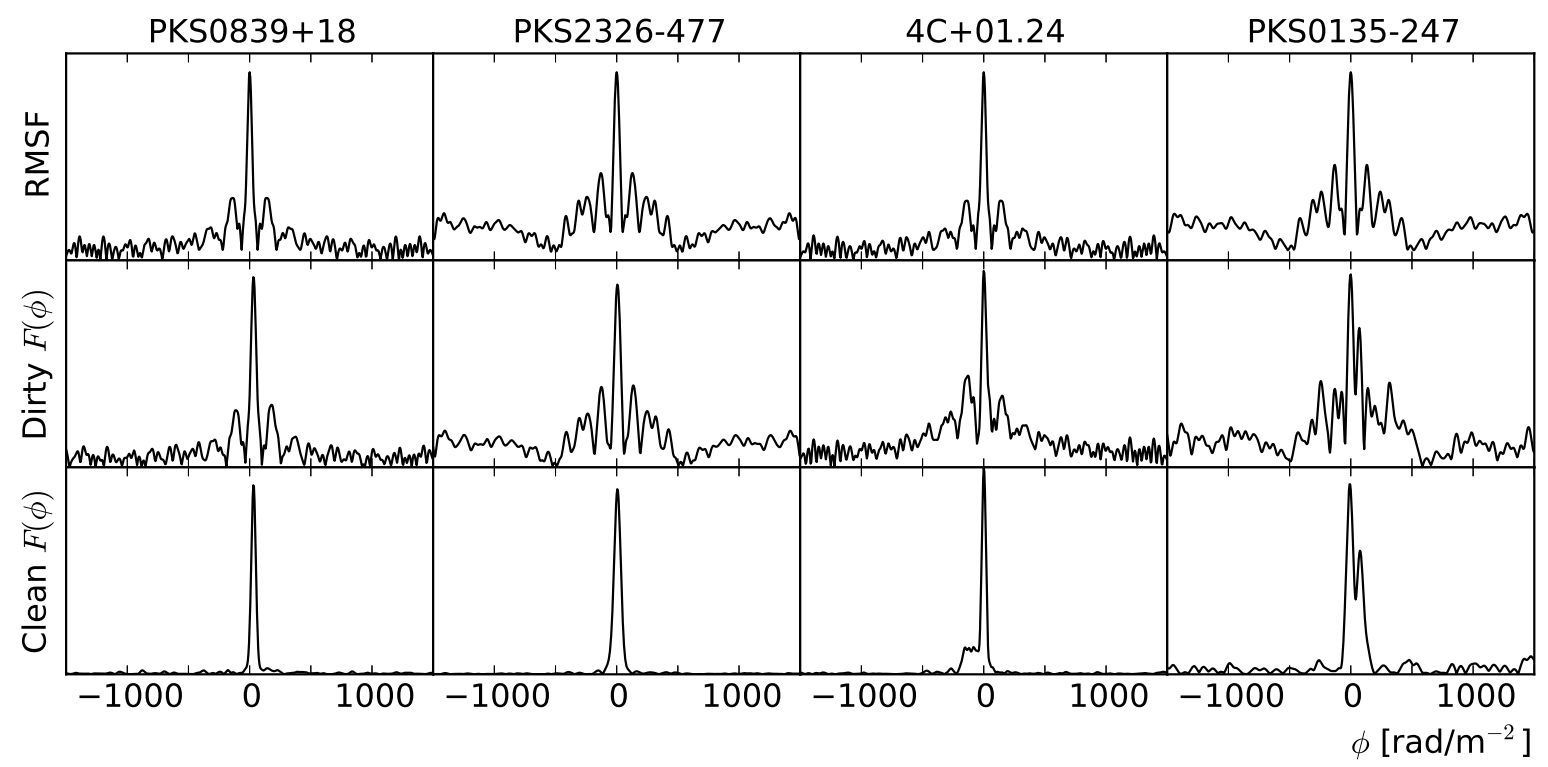

Figure 4. Effect of RM-CLEAN for four representative objects that have simple (first and second columns) and complex (third and fourth columns) FD distributions. The first and third column objects were observed with the VLA and the second and fourth column ones were observed with the ATCA. The first row represents the RMSF used by RM-CLEAN, the second row the dirty FD distribution, i.e. before RM-CLEAN is applied, and the third row the clean FD distribution after RM-CLEAN. The y-axes are in arbitrary units.

angle of a particular component at infinite frequency (see equation 5 above) while the radius is a measure of the amplitude that was plotted in Column (v). We obtain continuous curves since both $F(\phi)$ and $\psi(\phi)$ are continuous. The initial phase $\psi$ is defined between $-\pi / 2$ and $\pi / 2$ and so for plotting purposes, $\psi$ is multiplied by 2 .

The FD distribution $F(\phi)$ decomposes the observed flux density in $\phi$, i.e. it describes the amount of linearly polarized flux density which has undergone a certain amount of Faraday Rotation due to lying at a certain Faraday Depth. Thus, for a homogeneous screen lying in front of a simple source, the FD distribution would ideally be a delta function. An inhomogeneous screen would result in a broader or more complicated FD distribution because different parts of the background source would have passed through different Faraday depths $\phi$. In this work we are interested in extracting information on the inhomogeneity of foreground screens and therefore the FD distribution is what we are ultimately interested in.

However, from the FD distribution alone, we do not know if structure within $F(\phi)$ is caused by variations within an intervening system, somewhere along the line of sight, or by having a source which is itself Faraday thick. Sources are called Faraday thick if they have a range of $\phi$ due to magnetized plasma intrinsic to the sources. This can occur in two ways, either through internal Faraday dispersion or through differential Faraday Rotation (Sokoloff et al. (1998)). Internal Faraday dispersion is caused by intrinsic inhomogeneous screens. Effectively the source is composed of different sub-components each with their own $\phi$. Differential Faraday Rotation is caused if a source is extended along the line of sight such that flux which is emitted from the far side of the object undergoes more rotation than flux which is emitted from the near side.

To discriminate between intrinsic inhomogeneity effects and intervener inhomogeneity effects $\psi(\phi)$ can be very useful. As mentioned already in Section 1 the initial phase represents the average polarization angle of the flux density at a certain $\phi$. Thus if all the emission has the same origin then $\psi(\phi)$ should be constant, independent of how complex the FD distribution might be from the intervener system. If, however, $\psi(\phi)$ is not constant, then it can be concluded that the emission at the different $\phi$ must have different spatial origins within the source. Correspondingly, it becomes very likely (but not absolutely proven) that the variation of $\phi$ is also intrinsic to the source. The case of constant $\psi(\phi)$ corresponds to a radial locus of $\mathbf{F}$ in a polar representation, i.e. a linear feature extending out from the central origin. There are numerous cases of this in Figures 1-3 (e.g. PKS0839+18, $4 \mathrm{C}+13.46$ and $4 \mathrm{C}+05.64$ )

To illustrate a more complex example, consider again the case of a source (with intrinsic magnetic fields) that is extended along the line of sight. The emission coming from the far side of the source can be produced with different polarization angle than emission which is coming from the near side and therefore can have different initial phases. Eventually one would expect an ellipse like structure in the polar plots of the complex FD distribution in which the initial phase varies smoothly with the Faraday Depth. Analogously one would also get ellipselike structures if there are unresolved spatial polarization structures in the extended source as well as an spatially inhomogeneous FD screen in front of it. Examples of ellipses in Figures 1-3 are $4 \mathrm{C}+02.27$, OW-174 and $4 \mathrm{C}$ 05.62 .

More complex situations are also possible. A source consisting of a number of discrete components within the telescope beam, each with a very narrow range of $\phi$, will exhibit the corresponding number of peaks in $F(\phi)$ (in Column (v)) and radial spikes in $\mathbf{F}(\phi)$ (in Column (vi)) producing a complex amoeba-like structure in the latter. Examples are given by 4C-00.50, PKS1143-247 and 
PKS0506-61. On the other hand, a simple (but extended) background source that undergoes Faraday Rotation by a number of discrete $\phi$ within an inhomogeneous foreground screen would consist of multiple peaks in $F(\phi)$ (in Column (v)) but the radial spikes in $\mathbf{F}(\phi)$ (in Column (vi)) would be aligned. Interestingly, we see no such sources in our sample, a point to which we will return later.

Overall, we see that there is a wide variation in complexity of $F(\phi)$ and $\mathbf{F}(\phi)$ within our sample as shown in Figures 1-3. However, despite this clear variety, we also see that each object usually has a pronounced dominant Gaussian-like component in which a large fraction of the total flux density is contained. This is in agreement with the observations of low redshift quasars in Anderson et al. (2015). We will refer to this component as the "primary component" throughout this paper. All other significant components will be referred to as "secondary components". Roughly two third of our sample possess significant secondary components. Generally speaking, the structure of the FD distribution is broadly comparable between those sources observed at VLA and those observed at ATCA, except for the different width of the RMSF.

\section{PARAMETERS}

In this section we define all the parameters which will be used in Section 5 to quantify structures in polarization and in the FD distribution. Note that these parameters were defined blind to the presence of interveners, in the sense that we defined and measured them before linking the radio data with the optical spectra. The sole exception was $\sigma_{P C}$ for reasons which will become clear later. The parameter values of all objects are listed in Table 3 .

\subsection{Rotation Measure and $\phi_{\max }$}

As is clear in Figures 1-3, essentially every object (with the possible exception only of PKS2134+004, see below) has a FD distribution that is dominated by a single pronounced primary component at a well-defined $\phi_{\max }$. We define $\tilde{\phi}_{\max }$ as the peak position as observed, and $\phi_{\max }$ as the peak position after shifting the FD distribution by a uniform $\Delta \phi$ corresponding to an estimate of the Galactic foreground according to Oppermann et al. (2015).

It is therefore of interest to compare the $\phi_{\max }$ for each source with the $\mathrm{RM}_{\mathrm{Kron}}$ of Kronberg et al. (2008) used in Bernet et al. (2008). These were based on polarization angle measurements at just a few distinct frequencies. As discussed in Bernet et al. (2012), there are some caveats to this traditional way of measuring RM, especially if the sources are significantly depolarized. These problems are largely circumvented in RM Synthesis.

In Figure 5 we compare the primary component peak $\tilde{\phi}_{\max }$ of the FD distribution, before any Galactic RM correction, to the $\mathrm{RM}_{\mathrm{Kron}}$ which were used (similarly uncorrected) in Bernet et al. (2008). For about three quarters of the objects, the agreement between these quite independent measurements is very good with a random dispersion of roughly $8 \mathrm{rad} / \mathrm{m}^{2}$. For 12 objects there is a significant discrepancy. Many of the objects with strong $\left|\mathrm{RM}_{\mathrm{Kron}}\right|>50 \mathrm{rad} / \mathrm{m}^{2}$ are found to have rather smaller $\left|\tilde{\phi}_{\max }\right|$. There are three objects with very large discrepancies. The case of PKS2134+004 is interesting in that
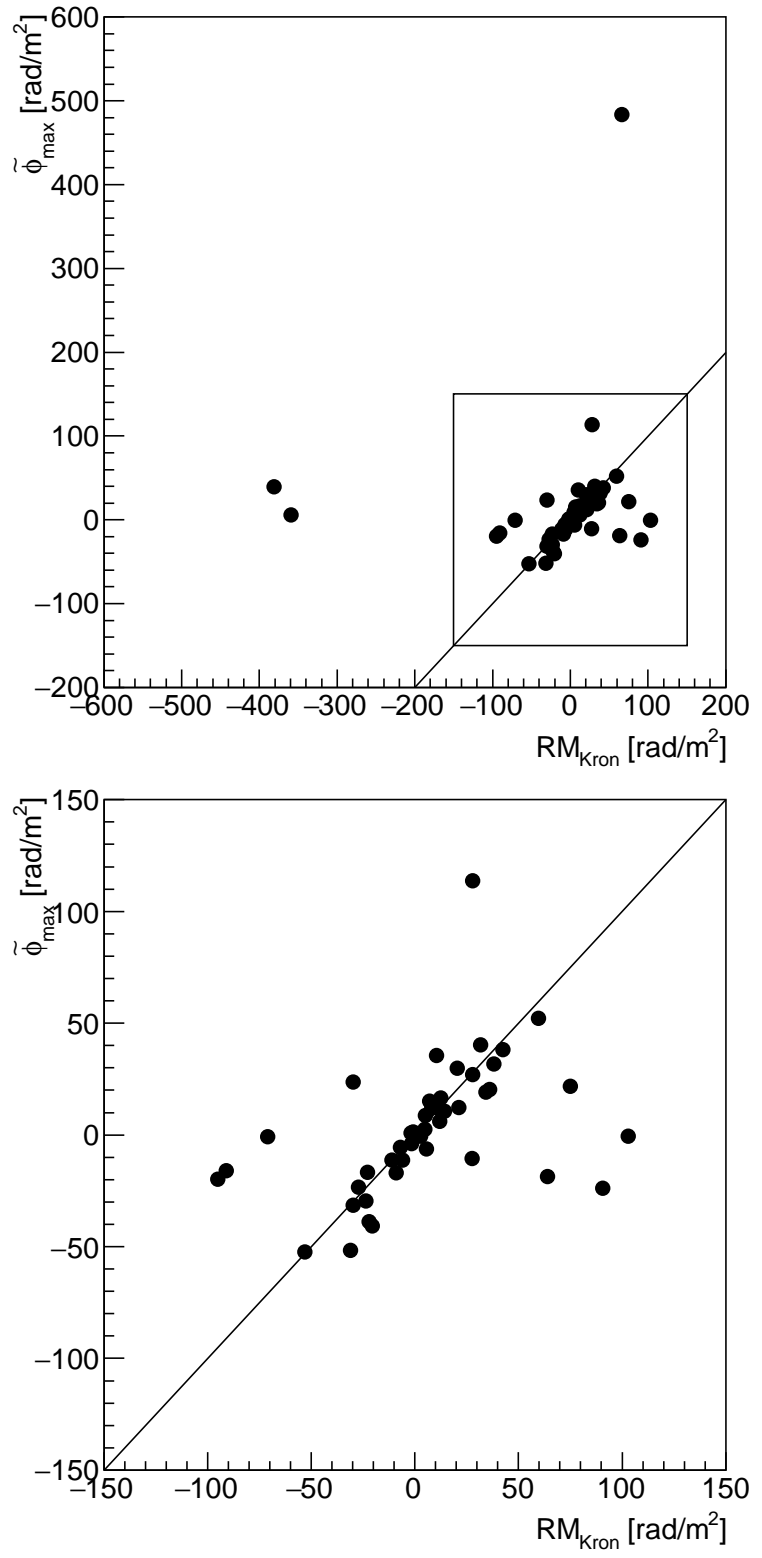

Figure 5. Comparison between $\mathrm{RM}_{\mathrm{Kron}}$ from Kronberg et al. (2008) and the Faraday Depth $\tilde{\phi}_{\max }$ at which the FD distribution before Galactic RM subtraction has its maximum for all objects. The lower panel is a zoomed in depiction of the boxed region in the upper panel.

the dominant peak is clearly at very high $\tilde{\phi}$. This source shows extraordinarily complex FD structures (Figure 3, Row 6). The position angle $\chi\left(\lambda^{2}\right)$ plot for this source emphasizes the difficulty of measuring an RM, for such a source, from sparsely sampled data. In the main, however, this comparison is reassuring that the original RM measurements used in Bernet et al. (2008) were meaningful.

\subsection{Depolarization}

Many years ago, Burn (1966) calculated the relation between depolarization and a dispersion in the Faraday Depth to be of the form

$$
\Pi\left(\lambda^{2}\right)=\Pi_{0} \exp \left(-2\left(\sigma_{\text {Burn }} \lambda^{2}\right)^{2}\right)
$$


Table 3

Depolarization and Faraday Depth Parameters

\begin{tabular}{|c|c|c|c|c|c|c|c|c|c|c|c|c|c|}
\hline Name & $\tilde{\phi}_{\max }{ }^{1}$ & $\phi_{\max }{ }^{1}$ & $\mathrm{DP}_{\text {Quart }}$ & $\mathrm{DP}_{\text {Half }}$ & $\mathrm{DP}_{\mathrm{Min} / \mathrm{Max}}$ & $\mathrm{DP}_{\mathrm{Min} / \mathrm{Max}}^{\prime}$ & $\sigma_{\text {Burn }}^{1}$ & $\sigma_{\text {Burn }}^{\prime}{ }^{1}$ & $\sigma_{\mathrm{FD}}{ }^{1,2}$ & $G^{2}$ & $C^{2}$ & Rank & $\sigma_{\mathrm{PC}}{ }^{1,2}$ \\
\hline PKS0130-17 & 52.2 & 43.7 & 0.902 & 0.779 & 0.976 & 0.976 & 25.17 & 25.04 & $103.5 / 52.7$ & $0.069 / 0.050$ & $0.111 / 0.044$ & 20 & $35.7 / 33.3$ \\
\hline PKS0135-247 & -6.3 & -17.6 & -0.392 & -0.286 & 0.851 & 0.197 & 0.00 & -3 & $55.4 / 116.9$ & $0.054 / 0.080$ & $0.366 / 0.444$ & 45 & $12.0 / 11.2$ \\
\hline PKS0139-09 & -19.7 & -29.6 & 0.775 & 0.713 & 0.873 & 0.873 & 28.30 & 27.98 & $50.8 / 117.7$ & $0.048 / 0.074$ & $0.058 / 0.181$ & 27 & $34.5 / 29.5$ \\
\hline $3 \mathrm{C} 057$ & -0.6 & -14.6 & -0.197 & -0.430 & 0.769 & 0.297 & -3 & -3 & 136.0 / 136.5 & $0.050 / 0.076$ & 0.140 / 0.230 & 24 & $16.8 / 17.4$ \\
\hline PKS0202-17 & 20.4 & 13.9 & -1.842 & -1.471 & 0.958 & 0.376 & ${ }_{-3}$ & 10.10 & $50.7 / 99.0$ & $0.042 / 0.066$ & $0.078 / 0.151$ & 16 & $21.2 / 23.7$ \\
\hline PKS0332-403 & -24.0 & -16.4 & 0.281 & 0.028 & 0.702 & 0.702 & 9.76 & 9.26 & $167.6 / 156.7$ & 0.117 / 0.102 & $0.391 / 0.377$ & 43 & $28.2 / 20.9$ \\
\hline PKS0402-362 & -0.7 & 12.4 & -1.085 & -0.289 & 0.897 & 0.399 & $-^{3}$ & 9.28 & $42.6 / 94.0$ & $0.038 / 0.073$ & $0.093 / 0.204$ & 9 & $10.6 / 25.3$ \\
\hline PKS0422-380 & 39.1 & 55.3 & -1.243 & -0.661 & 0.945 & 0.542 & $-^{3}$ & -3 & $25.7 / 89.0$ & $0.026 / 0.063$ & $0.000 / 0.251$ & 13 & $16.2 / 9.1$ \\
\hline PKS0426-380 & 38.1 & 55.0 & 0.696 & 0.641 & 0.850 & 0.850 & 19.69 & 18.33 & $84.5 / 53.2$ & $0.055 / 0.050$ & $0.116 / 0.355$ & 21 & $21.7 / 8.7$ \\
\hline PKS0506-61 & 113.7 & 179.9 & 0.790 & 0.609 & 0.912 & 0.912 & 24.64 & 18.24 & $80.2 / 72.4$ & $0.073 / 0.068$ & $0.258 / 0.030$ & 48 & $31.6 / 58.0$ \\
\hline PKS0839+18 & 31.8 & 44.4 & 0.117 & 0.082 & 0.352 & 0.337 & 3.50 & 4.48 & $43.4 / 41.2$ & $0.030 / 0.026$ & $0.097 / 0.076$ & 17 & $7.8 / 6.3$ \\
\hline $4 \mathrm{C}+01.24$ & 0.8 & 23.6 & -0.247 & -0.138 & 0.634 & 0.000 & $-^{3}$ & -3 & $70.5 / 64.0$ & $0.051 / 0.042$ & $0.314 / 0.240$ & 46 & $3.4 / 7.2$ \\
\hline $4 \mathrm{C}+02.27$ & -11.2 & 8.1 & 0.282 & 0.179 & 0.510 & 0.510 & 5.76 & 5.71 & $22.6 / 24.8$ & $0.022 / 0.021$ & $0.007 / 0.042$ & 8 & $12.6 / 4.6$ \\
\hline $\mathrm{OK}+186$ & -3.9 & 1.2 & 0.190 & 0.098 & 0.368 & 0.368 & 4.12 & 4.81 & $32.8 / 28.4$ & $0.026 / 0.023$ & $0.072 / 0.052$ & 11 & $9.1 / 7.5$ \\
\hline $4 C+19.34$ & 35.6 & 28.1 & 0.638 & 0.621 & 0.917 & 0.917 & 12.77 & 15.55 & $36.3 / 48.1$ & $0.035 / 0.044$ & $0.053 / 0.111$ & 26 & $24.4 / 25.7$ \\
\hline $4 \mathrm{C}+06.41$ & 10.5 & -11.0 & 0.332 & 0.146 & 0.536 & 0.536 & 5.80 & 5.76 & $91.5 / 91.6$ & $0.054 / 0.051$ & $0.217 / 0.194$ & 28 & $9.1 / 8.1$ \\
\hline $3 \mathrm{C} 245$ & 23.7 & 6.8 & 0.879 & 0.715 & 0.922 & 0.922 & 14.09 & 14.33 & $37.9 / 36.7$ & 0.033 / 0.029 & $0.052 / 0.034$ & 15 & $19.9 / 17.4$ \\
\hline $4 \mathrm{C}+20.24$ & -38.8 & -56.6 & -1.467 & 0.243 & 0.973 & 0.676 & -3 & 9.56 & $47.0 / 35.4$ & 0.040 / 0.034 & 0.070 / 0.033 & 22 & $25.4 / 25.7$ \\
\hline PKS1111+149 & 12.5 & 5.0 & 0.381 & 0.376 & 0.808 & 0.808 & 7.68 & 7.64 & $34.9 / 34.3$ & $0.028 / 0.028$ & $0.090 / 0.083$ & 14 & $12.0 / 12.8$ \\
\hline PKS1127-14 & 40.3 & 40.4 & 0.394 & 0.262 & 0.505 & 0.505 & 11.41 & 4.24 & $70.2 / 56.0$ & $0.052 / 0.045$ & $0.197 / 0.181$ & 34 & $2.3 / 19.0$ \\
\hline PKS1143-245 & -0.5 & -18.0 & 0.259 & 0.425 & 0.884 & 0.884 & 15.52 & 15.19 & $82.3 / 279.3$ & $0.068 / 0.095$ & $0.409 / 0.375$ & 47 & $15.6 /-^{4}$ \\
\hline PKS1157+014 & 2.5 & -4.8 & -0.016 & 0.219 & 0.804 & 0.774 & 4.58 & 8.46 & $62.2 / 65.2$ & $0.047 / 0.047$ & 0.149 / 0.173 & 19 & $17.1 / 17.2$ \\
\hline $4 \mathrm{C}+13.46$ & 6.1 & 2.8 & 0.580 & 0.320 & 0.6 & 0.683 & 9.46 & 9.31 & $23.7 / 92.1$ & $0.021 / 0.032$ & $0.027 / 0$. & 4 & $8.0 / 8.5$ \\
\hline $4 \mathrm{C}-02.55$ & -18.7 & -19.6 & 0.549 & 0.515 & 0.877 & 0.877 & 28.92 & 28.96 & 113.0 / 106.8 & $0.052 / 0.051$ & $0.350 / 0.151$ & 41 & $12.4 / 28.7$ \\
\hline PKS1244-255 & -29.5 & -47.1 & -2.787 & -1.189 & 1.000 & 1.000 & 0.00 & 14.94 & 46.4 / 46.4 & $0.041 / 0.041$ & $0.101 / 0.064$ & 32 & $17.1 / 22.6$ \\
\hline $\mathrm{ON}+187$ & 15.1 & 13.1 & -0.349 & -0.237 & 1 & 0.155 & 0.00 & -3 & 133.0 / 128.3 & 0.085 / 0.081 & 0.379 / 0.367 & 44 & 6.9 / 6.4 \\
\hline $4 \mathrm{C}-00.50$ & 16.5 & 10.8 & 0.615 & 0.634 & 4 & 0.864 & 21.83 & 21.69 & $44.7 / 48.2$ & 0.036 / 0.041 & $0.257 / 0$. & 39 & $9.6 / 17.8$ \\
\hline $4 \mathrm{C}+19.44$ & 8.8 & 10.2 & -0.633 & -0.093 & 3 & 0.280 & 0.00 & 5.29 & $37.2 / 56.6$ & $0.027 / 0.036$ & 0.070 / 0 . & 18 & $10.8 / 12.5$ \\
\hline $3 \mathrm{C} 298$ & -40.6 & -47.6 & 0.918 & 0.483 & 0.5 & 0.985 & 79.93 & 78.68 & $35.9 / 132.8$ & 0.030 / 0.122 & $0.306 / 0.599$ & 42 & $6.7 / 28.6$ \\
\hline PKSB1419-272 & -17.1 & -52.8 & 0.669 & 0.487 & 0.832 & 0.832 & 10.63 & 11.29 & 33.7 / 42.0 & $0.031 / 0.033$ & $0.124 / 0.104$ & 33 & $12.7 / 16.6$ \\
\hline OQ+135 & 12.3 & 3.8 & 0.485 & 0.109 & 0.6 & 0.666 & 7.64 & 7.29 & $69.6 / 56.5$ & $0.030 / 0.040$ & $0.147 / 0.248$ & 31 & $6.5 /-4$ \\
\hline $4 \mathrm{C}-05.62$ & 1.3 & -2.9 & 0.337 & 0.190 & 0.425 & 0.425 & 6.20 & 6.38 & $27.7 / 40.7$ & $0.024 / 0.023$ & $0.067 / 0.035$ & 2 & $10.4 / 7.9$ \\
\hline 4C-05.64 & -16.8 & -25.2 & 0015 & -0.058 & 0773 & 0166 & -3 & -3 & 139.5 / 136.3 & $0.037 / 0.035$ & $0.226 / 0.214$ & 25 & $-4 /-4$ \\
\hline $4 \mathrm{C}+05.64$ & -10.6 & -5.9 & .030 & 0.039 & 9 & 79 & 1.79 & 0.00 & $32.8 / 24.0$ & $0.024 / 0.021$ & $0.064 / 0.045$ & 10 & $2.5 / 2.3$ \\
\hline PKS1615+029 & 21.9 & 30.4 & & 0.226 & & & 7.44 & 6.40 & $126.3 / 123.3$ & $0.050 / 0.050$ & $0.235 / 0.261$ & 30 & $3.4 / 6.2$ \\
\hline OW-174 & -31.5 & 44.5 & 0.858 & 0.713 & .502 & .982 & 13.39 & 13.31 & $44.2 / 43.5$ & $0.035 / 0.034$ & 0.110 / 0.113 & 35 & $18.6 / 17.5$ \\
\hline OX-325 & 27.0 & 25.7 & -0.249 & -0.086 & 0.461 & 0.241 & -3 & -3 & $27.9 / 59.3$ & $0.024 / 0.040$ & $0.031 / 0.167$ & 7 & $13.3 / 10.1$ \\
\hline PKS2134+004 & 483.5 & 524.3 & & & & 96 & 42.02 & 39.44 & $207.4 / 197.5$ & $0.182 / 0.136$ & $0.396 / 0$ & 49 & $63.3 / 47.2$ \\
\hline OX-173 & 18.3 & 28.4 & 0.278 & 0.208 & 0.512 & 0.512 & 7.39 & 3.88 & $87.2 / 30.4$ & $0.054 / 0.030$ & $0.287 / 0.022$ & 37 & $8.2 / 14.4$ \\
\hline $4 \mathrm{C}+6.69$ & 29.8 & 96.2 & 0.527 & 0.225 & 0.932 & 0.932 & 7.13 & 6.50 & $610.2 / 547.3$ & $0.036 / 0.038$ & 0.229 / 0.193 & 5 & $12.9 / 15.0$ \\
\hline OX-192 & 11.9 & 17.1 & -0.408 & -0.230 & 0.704 & 0.340 & -3 & 7.30 & 29.0 / 30.3 & $0.028 / 0.029$ & 0.019 / 0.020 & 6 & $10.5 / 13.2$ \\
\hline PKS2204-54 & 12.2 & 13.7 & -0.727 & -0.103 & 0.635 & 0.127 & -3 & -3 & $102.9 / 49.7$ & $0.060 / 0.042$ & $0.206 / 0.131$ & 23 & $15.4 / 8.9$ \\
\hline & -5.5 & & & & & & 3.56 & 2.39 & $63.1 / 48.1$ & $0.046 / 0.034$ & $0.197 / 0.143$ & 38 & $8.9 / 5.5$ \\
\hline PKS2223-05 & -23.5 & -10.3 & 0.178 & 0.056 & 0.410 & 0.410 & 3.96 & 6.45 & $25.5 / 25.0$ & $0.022 / 0.022$ & $0.028 / 0.038$ & 1 & $10.6 / 7.5$ \\
\hline PKS2227-08 & -11.0 & -11.7 & -0.025 & -0.053 & 0.511 & 0.349 & 1.80 & -3 & $62.0 / 58.4$ & $0.048 / 0.046$ & 0.133 / 0.139 & 29 & $9.4 / 6.9$ \\
\hline-11.69 & -52.4 & -16.5 & 0.361 & & & & 7.26 & 7.13 & $53.9 / 81.5$ & $0.034 / 0.055$ & $0.155 / 0.211$ & 36 & $8.1 / 8.9$ \\
\hline PKS2243-123 & -15.9 & -2.5 & 0.273 & 0.138 & 0.654 & 0.654 & 9.07 & 6.54 & $98.8 / 61.6$ & $0.086 / 0.044$ & $0.344 / 0.064$ & 40 & $12.8 / 22.4$ \\
\hline & -51.7 & -4.4 & -0.0 & & & & 1.10 & 5.04 & $44.1 / 42.8$ & 0.030 / 0.029 & 0.079 / 0 & 12 & $12.6 / 10.7$ \\
\hline PKS2326-477 & 5.9 & -8.7 & 0.180 & 0.226 & 0.521 & 0.521 & 6.63 & 9.44 & $50.3 / 96.2$ & $0.038 / 0.083$ & $0.066 / 0.353$ & 3 & $15.4 / 9.3$ \\
\hline
\end{tabular}

1 in units of $\left[\mathrm{rad} / \mathrm{m}^{2}\right]$. The $\tilde{\phi}_{\max }$ parameter is before and the $\phi_{\max }$ parameter after Galactic foreground subtraction.

2 The second numbers are the values when fractional $u$ and $q$ is utilized for RM Synthesis (cf. Section 2.4).

3 The fit has failed to converge or unphysical values has been obtained.

${ }^{4} \sigma_{\text {RMSF }}$ has been larger than $\sigma_{\text {fit }}$. 
where $\Pi\left(\lambda^{2}\right)$ is the polarization fraction as a function of $\lambda^{2}, \Pi_{0}$ is the intrinsic polarization fraction before the Faraday depolarization, which is assumed to be constant with $\lambda$, and $\sigma_{\text {Burn }}$ is the standard deviation of a Gaussian FD distribution. Subsequently, various modifications to this simple model have been proposed to better fit observations (Tribble (1991), Rossetti et al. (2008), Bernet et al. (2012)).

All these models produce a monotonic decrease of $\Pi\left(\lambda^{2}\right)$ with $\lambda$. The measured polarization of many of our sources, however, clearly shows a more complex structure in $\Pi\left(\lambda^{2}\right)$. We will explore possible reasons for this in Section 5.4. An extensive overview of depolarization due to Faraday screens can be found in the appendix of Farnes et al. (2014).

We want to test the suggestion in Bernet et al. (2012) that intervening MgII absorption along the line of sight also produces detectable depolarization effects. We therefore need to quantify the depolarization in the radio spectra. However, the variety and complexity of the polarization spectra (Column (iii) in Figure 1-3) makes it impossible to represent the depolarization with a single parameter. We therefore defined several parameters. Although some of these may appear somewhat ad hoc, this was done blind to the presence of interveners.

We first define $\mathrm{DP}_{\text {Quart }}$ and $\mathrm{DP}_{\text {Half }}$ as

$$
\mathrm{DP}_{\text {Quart }, \text { Half }}=1-\frac{\Pi_{2}}{\Pi_{1}}
$$

where $\Pi_{1}$ is the median $\Pi$ in the first quarter of the observed $\lambda^{2}$ band, i.e. in the short wavelength end, and $\Pi_{2}$ the median $\Pi$ in the last quarter for $\mathrm{DP}_{\text {Quart }}$, i.e. in the long wavelength end. For $\mathrm{DP}_{\mathrm{Half}}, \Pi_{1}$ is the median $\Pi$ in the first half of the observed $\lambda^{2}$ band and $\Pi_{2}$ the median $\Pi$ in the other half. By definition $\mathrm{DP}_{\text {Quart }}$ and $\mathrm{DP}_{\text {Half }}$ yield negative values for re-polarizing sources.

We also define $\mathrm{DP}_{\mathrm{Min} / \mathrm{Max}}$ as

$$
\mathrm{DP}_{\text {Min } / \text { Max }}=1-\frac{\Pi_{\min }}{\Pi_{\max }}
$$

where $\Pi_{\min }$ and $\Pi_{\max }$ are the minimum and maximum observed $\Pi$, respectively wherever they occur in the observed $\lambda$ range. We also define $\mathrm{DP}^{\prime}{ }_{\text {Min/Max }}$, which is similar to $\mathrm{DP}_{\text {Min/Max }}$ except that $\Pi_{\min }$ is the minimum polarization at a longer wavelength than the maximum $\Pi_{\text {max }}$. $\mathrm{DP}_{\text {Quart }}, \mathrm{DP}_{\text {Half }}, \mathrm{DP}_{\text {Min } / \text { Max }}$ and $\mathrm{DP}^{\prime}{ }_{\text {Min/Max }}$ are completely phenomenological parameters.

Lastly we introduce $\sigma_{\text {Burn }}$ and $\sigma_{\text {Burn }}^{\prime}$ as two further depolarization parameters. The $\sigma_{\text {Burn }}$ parameter is defined as in equation 8 and is obtained by fitting this relation to $\Pi\left(\lambda^{2}\right)$ over the whole observed wavelength range while the $\sigma_{\text {Burn }}^{\prime}$ parameter is obtained by only fitting $\Pi\left(\lambda^{2}\right)$ at wavelengths longer than $\Pi_{\max }$. The latter is motivated by the conjecture that depolarization might be mainly caused by a simple dispersion in Faraday Depth while other more complex behavior of the polarization structure might be caused by other processes. The fits are carried out by using the method of least squares. Although $\sigma_{\text {Burn }}$ and $\sigma_{\text {Burn }}^{\prime}$ might seem like the most appropriate parameters if the FD distribution was Gaussian, they might be less appropriate for the more complex polarization curves. The values of the six depolarization parameters for each object are listed in Table 3.

\subsection{FD distribution}

As with depolarization, it is not trivial to identify the most suitable parameters to characterize the structure in the FD distributions due to the variety of the observed distributions (see Figures 1-3, Column (v)). As in the previous section, we approached this problem by defining several parameters. To preserve objectivity, we again defined all the parameters in this section blind to the presence of interveners in the sources.

An important point is that, because RM Synthesis behaves like a Fourier Transform, random "white" noise in the input $\mathrm{Q}$ and $\mathrm{U}$ spectra will produce a non-zero contribution to the FD distribution $F(\phi)$ that should be independent of $\phi$ and extends out to the $\phi$ corresponding to the spectral resolution of the input data, in our case $\pm 1500 \mathrm{rad} / \mathrm{m}^{2}$. In practice this noise contribution to the FD distribution will itself have structure due to noise in the data. To avoid including this spurious contribution into our characterization of the structure in the FD distribution, the following thresholding scheme was implemented. We first apply an iterative sigma-clipping algorithm (clipping at $3 \sigma$ ) to $F(\phi)$ over the whole $\pm 1500 \mathrm{rad} / \mathrm{m}^{2}$ range to estimate the background mean and standard deviation of $F(\phi)$ that arises from this noise. We then identify real signals in $F(\phi)$ as being those regions which exceed this mean plus $5 \sigma$, in the sense that we can be reasonably confident that $F(\phi)$ in these regions is not coming from noise. These are the parts of $\phi$ space that we want to use to characterize the FD distribution. This threshold is indicated by the magenta lines in Column (v) of Figures 1-3. For each such region above the threshold, we then extend in each direction out to the $\phi$ at which $F(\phi)$ first crosses the mean background level. All other regions are set to zero, producing a thresholded $\hat{F}(\phi)$ function that can then be parameterized as described below.

The first parameter is simply the second moment of the modified FD distribution $\hat{F}(\phi)$

$$
\sigma_{\mathrm{FD}}^{2}=\frac{\int \hat{F}(\phi)\left(\phi-\mu_{\mathrm{FD}}\right)^{2} d \phi}{\int \hat{F}(\phi) d \phi}
$$

where $\mu_{\mathrm{FD}}$ is the usual first-moment of the FD distribution. Because RM Synthesis yields a discrete FD distribution at equidistant $\phi_{i}$, this integral is conveniently done as a sum over the non-zero data points. This parameter has also been used in Anderson et al. (2015).

The second parameter is the reversed Gini coefficient $G$. The reversed Gini coefficient is simply defined (for our equidistant data points) as

$$
G=1-\frac{1}{2 \mu_{\mathrm{FD}} n(n-1)} \sum_{i}^{n} \sum_{j}^{n}\left|\hat{F}\left(\phi_{i}\right)-\hat{F}\left(\phi_{j}\right)\right| .
$$

where the sum is carried out over the full range of $\phi$ so that $n$ is the same for all objects. This means that objects can be directly compared to each other, even though the absolute value of $G$ depends on the range of $\phi$ (i.e. on $n$ ). The Gini coefficient is generally used to measure the concentration of a distribution, e.g. in economics to measure the concentration of wealth within a population (Gini (1912)) or in astronomy to measure the luminosity concentration in a galaxy (Abraham \& van Bergh (2003)). 


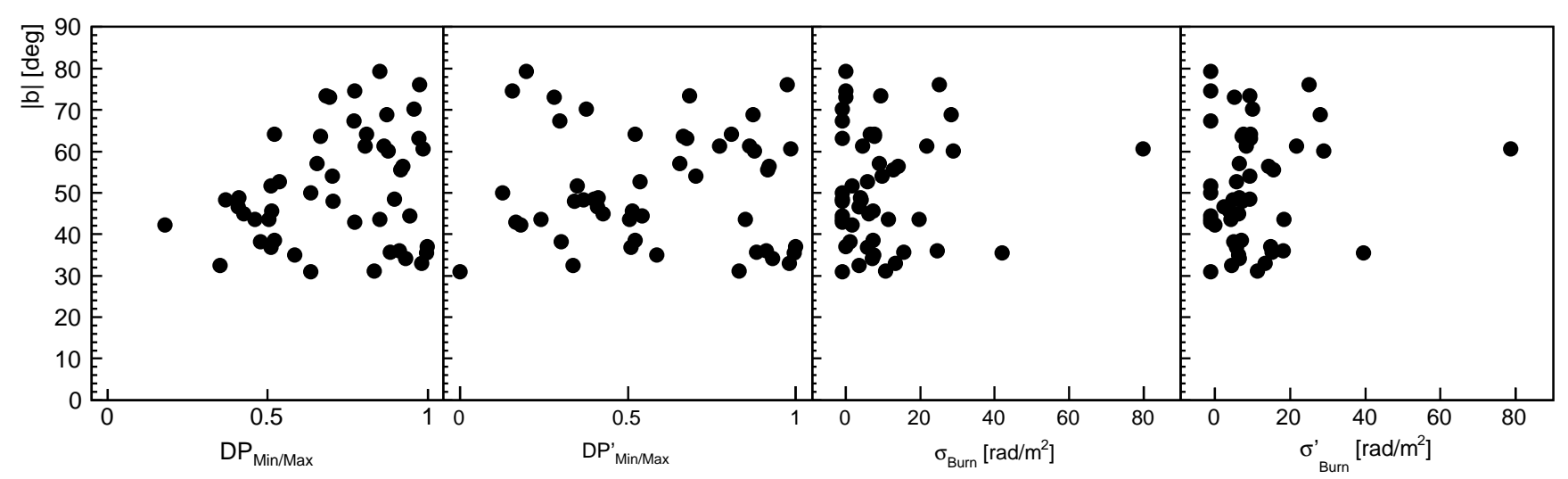

Figure 6. Comparison of absolute Galactic latitude $|b|$ to the depolarization parameters $\mathrm{DP}_{\mathrm{Min} / \mathrm{Max}}$ (left), DP ${ }_{\text {Min/Max }}$ (middle left), $\sigma_{\mathrm{Burn}}$ (middle right) and $\sigma_{\mathrm{Burn}}^{\prime}$ (right), respectively. Objects at high $|b|$ seem to have enhanced $\mathrm{DP}_{\mathrm{Min} / \text { Max }}$. There is no obvious trend for $\mathrm{DP}_{\text {Min/Max }}^{\prime}, \sigma_{\text {Burn }}$ and $\sigma_{\text {Burn }}^{\prime}$ with $|b|$.

$G$ is bounded between 0 and 1 whereby 1 means equal distribution and 0 means total concentration. We choose the reversed Gini coefficient because we want to maintain the convention that non-concentrated FD distributions yield large parameters. In practice, since many sources are dominated by a single component, $G$ correlates quite well with $\sigma_{\mathrm{FD}}$.

We also introduce a "coverage parameter" $C$. This parameter is motivated by the suggestion that interveners may only partially cover the source (Rossetti et al. (2008), Mantovani et al. (2009), Bernet et al. (2012)). In this scenario, we could interpret that the primary component in the FD distribution represents the flux which is not covered by the intervening screen. We model the primary component by fitting a Gaussian to it, using the method of least squares, and subtracting that fit from $\hat{F}(\phi)$ distribution to yield $\hat{F}_{\text {cov }}(\phi)$. Our coverage parameter is then determined as

$$
C=\frac{\int \hat{F}_{\mathrm{cov}}(\phi) d \phi}{\int \hat{F}(\phi) d \phi} .
$$

We obtain values of $C$ up to around $40 \%$ in our sample.

With the parameters above we have attempted to describe the variety of complexity that we clearly can see. As a final subjective classification we ranked the objects in order of perceived complexity. Two of us (KSK and SJL) independently ranked the FD distributions. Despite the obvious subjectivity, these rankings agreed well, except for the roughly one third of the sources which have strikingly simple and therefore very similar FD distributions. However, the Kolmogorov Smirnov (KS) test which we will be using throughout this paper, is relatively robust against permutations within only the low ranked fraction of the objects. This method is to be regarded mostly as a test whether the quantitative parameters we used do indeed represent the perceived complexity. This subjective classification was again done blind to the presence of MgII absorption.

For reasons which will become clearer later (cf. Section 5.4) we introduce one more parameter $\sigma_{\mathrm{PC}}$, which has been defined a posteriori. The $\sigma_{\mathrm{PC}}$ parameter is obtained by fitting a Gaussian to the primary component. The range for this fit is where the FD distribution $F(\phi)$ stops declining on either side of the primary component.

To correct for the intrinsic spread function (RMSF) in $\phi$ that arises from the finite wavelength band-width of the radio data, which is different for the VLA and ATCA observations, we subtract in quadrature the predicted RMSF from the fitted $\sigma_{\text {fit }}$ as follows:

$$
\sigma_{\mathrm{PC}}=\sqrt{\sigma_{\mathrm{fit}}^{2}-\sigma_{\mathrm{RMSF}}^{2}}
$$

where $\sigma_{\mathrm{RMSF}}$ is defined in equation 7 . The obtained values of all five FD distribution parameters for each object are listed in Table 3.

\section{EFFECTS WITH GALACTIC LATITUDE}

As discussed earlier, Faraday Rotation can occur anywhere along the line of sight, including within the Galaxy, and it is therefore of interest to see if any of the parameters measured in the previous section correlate with the Galactic latitude $b$. We will discuss in detail the depolarization parameters $\mathrm{DP}_{\mathrm{Min} / \mathrm{Max}}, \mathrm{DP}_{\text {Min/Max }}^{\prime}, \sigma_{\mathrm{Burn}}$ and $\sigma_{\text {Burn }}^{\prime}$ and $\phi_{\max }$. These are most meaningful because they should be independent on the instrument (VLA or ATCA) other than the FD distribution parameters such as $\sigma_{\mathrm{FD}}$ and $\sigma_{\mathrm{PC}}$. However, we have checked these two parameters as well and have not found any obvious trends with $b$.

Figure 6 shows the four depolarization parameters plotted against $|b|$. Reassuringly, there is very little correlation in these plots except for an apparent absence of sources in the left-most panel with low $\mathrm{DP}_{\mathrm{Min} / \mathrm{Max}}$ at high Galactic latitudes. Recall that low $\mathrm{DP}_{\mathrm{Min} / \mathrm{Max}}$ corresponds to low depolarization, or more specifically a flat polarization in wavelength. We have no explanation as to why sources at high Galactic latitude would have more complex polarization. We note that the effect is not seen in the similar $\mathrm{DP}_{\text {Min/Max }}^{\prime}$ parameter (in the adjacent panel). We are confident that this possible effect in no way drives the results which will be quoted later(Section 5.2). In fact, if we exclude all the high Galactic latitude sources with $|b|>55^{\circ}$ from the analysis, the formal significance of the association between $\mathrm{DP}_{\mathrm{Min} / \mathrm{Max}}$ and the presence of intervening MgII absorption in the remaining sources actually increases. 


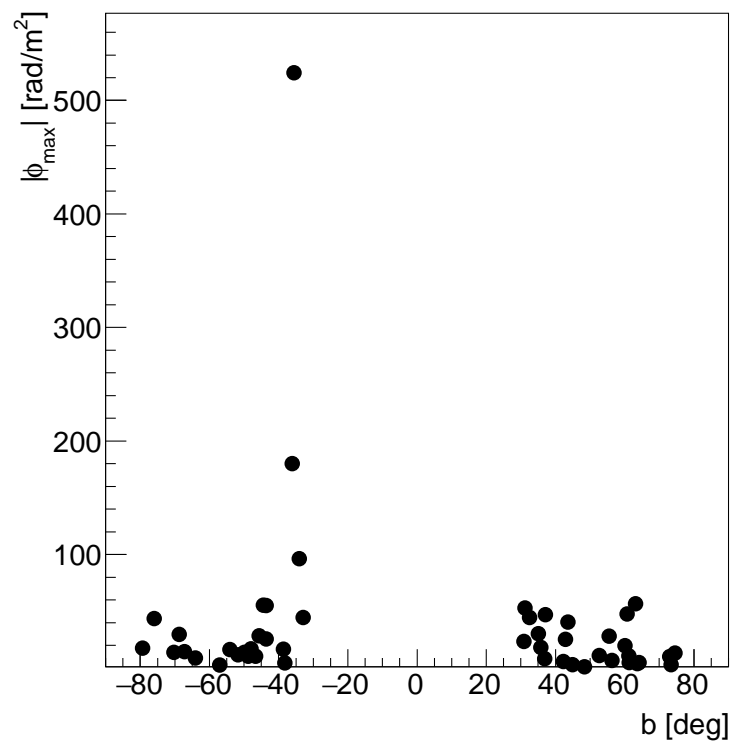

Figure 7. Comparison of Galactic latitude $b$ to the maximum peak position of the FD distribution $\phi_{\max }$ after Galactic RM subtraction. There is no overall trend of $\phi_{\max }$ with $b$ except for the two or three outlying objects with excessively large $\phi_{\max }$ which are all at around $|b| \approx 35^{\circ}$.

Turning to $\phi_{\max }$, Figure 7 shows this parameter, after our correction for the Galactic RM, according to the Oppermann et al. (2015) estimates, as a function of $b$. It is clear that the three highest values of $\left|\phi_{\max }\right|>80 \mathrm{rad} / \mathrm{m}^{2}$ are all found within a small range of $b$ around $b \approx-35^{\circ}$, and are therefore suspect. They are spread in Galactic longitude (at $55^{\circ}$, at $64^{\circ}$ and at $277^{\circ}$ ) and are in regions of not very high Galactic RM in the maps provided by Oppermann et al. (2015). Curiously, however, two of the three are at local maxima. Regardless of the origin, these three values are clearly suspect. These three sources also have intervening MgII absorption systems. Therefore they are excluded from the $\phi_{\max }$ analysis in Section 5.1.

\section{RESULTS}

\subsection{Rotation Measure and $\phi_{\max }$}

In Bernet et al. (2008), it was claimed that there was an association between higher $|\mathrm{RM}|$ values and the presence of strong (rest equivalent width above $0.3 \AA$ ) MgII absorption in the optical spectrum. This was based on a KS test on the distribution of $|\mathrm{RM}|$ for sources with and without such absorption. Here we reproduce this analysis but now using our new $\left|\phi_{\max }\right|$ instead of $\left|\mathrm{RM}_{\mathrm{Kron}}\right|$. $\left|\phi_{\max }\right|$ is the modulus of the Faraday Depth at which the FD distribution peaks, shifted by the Oppermann et al. (2015) estimates of the Galactic contribution to $\phi$. We use the one-tailed Kolmogorov-Smirnov to test the hypothesis that objects with MgII absorption along the lines of sight have enhanced $\left|\phi_{\max }\right|$ (i.e. our null hypothesis is that there is no such association or that clean lines of sight have enhanced $\left.\left|\phi_{\max }\right|\right)$. Recall that objects with $\phi_{\max }>80 \mathrm{rad} / \mathrm{m}^{2}$, i.e. PKS0506-61, PKS2134+004 and $4 \mathrm{C}+6.69$, are excluded from the analysis since we believe they are strongly affected by Galactic effects (see Section 4).

Unlike Bernet et al. (2008), we adopt a weaker absorp-

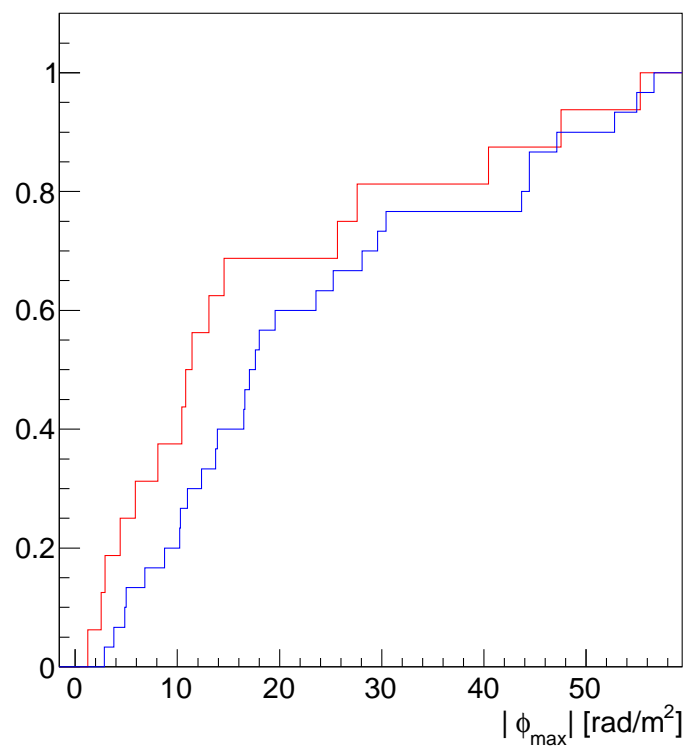

Figure 8. Empirical distribution function (EDF) of $\left|\phi_{\max }\right|$ for objects without (red) and with (blue) interveners. The one-tailed KS test yields $p=17 \%$.

tion cut at $W_{0}=0.1 \AA$ and get $p=17 \%$, represented in Figure 8. We will use this cut throughout the remainder of the paper because this cut turns out to be more significant later on when we analyze the distribution structure of Faraday Depth indicating that also weak absorbers affect the FD distribution. However, also using the same equivalent width cut at $0.3 \AA$ in $\mathrm{MgII}$ absorption, we do not see a signal and obtain a p-value of $23 \%$. Using fractional $q$ and $u$ for RM Synthesis as discussed in Section 2.4 makes effectively no difference to the significance level of this test for $\left|\phi_{\max }\right|$.

With our new data, the correlation of MgII with $\left|\phi_{\max }\right|$ is less significant than found with RM in Bernet et al. (2008). They found a significance level of $92.2 \%$ in a two-tailed KS test, without even correcting for Galactic RM.

This difference may be partly due to the fact that we are only analyzing a subset of their sample. On the other hand, it could also reflect differences between the use of $\phi_{\max }$ derived from RM Synthesis (as here) and the use of single RM values that are derived from individual sparsely-sampled polarization data (as in Bernet et al. (2008)). Indeed, when we do the KS test with $\mathrm{RM}_{\mathrm{Kron}}$ for our set of sample (excluding PKS0506-61, PKS2134+004 and $4 \mathrm{C}+6.69$ as with our $\phi_{\max }$ analysis) we obtain a somewhat stronger result with $p=13 \%$. This stronger significance is driven mostly by the objects with $\left|\mathrm{RM}_{\text {Kron }}\right|>50 \mathrm{rad} / \mathrm{m}^{2}$ which is where we see discrepancies between $\phi_{\max }$ and $\mathrm{RM}_{\mathrm{Kron}}$, as mentioned in Section 3.1. This difference between $\phi_{\max }$ and $\mathrm{RM}_{\mathrm{Kron}}$ will be explored further in a future paper.

Prompted by the statistical editor, we also have performed the Anderson-Darling (AD) 2-sample test for all our KS test results (here and later). It turns out that the $\mathrm{AD}$ test results are always slightly more significant but altogether consistent with the KS test results. Therefore we remain with the $\mathrm{KS}$ test throughout this paper.

It should be noted that the sources with intervening absorption systems are generally at slightly higher redshifts 


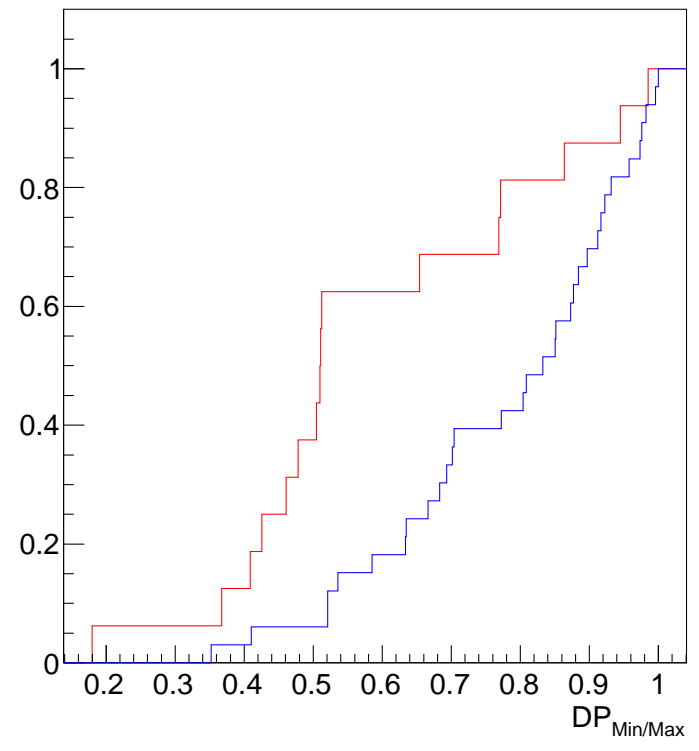

Figure 9. EDF of the depolarization parameter $\mathrm{DP}_{\mathrm{Min} / \mathrm{Max}}$ for objects without (red) and with (blue) interveners. The one-tailed KS test yields $p=0.1 \%$.

(difference of around 0.2 in their respective medians) and so the simple dependence of $\phi$ on wavelength would produce, all other things being equal, a lower observed dispersion for the more distant systems, i.e. opposite to what is seen in Figure 8.

\subsection{Depolarization}

We now look for correlations between the depolarization parameters and the presence of MgII absorption lines along the line of sight. As before, we apply the one-tailed KS test with the null hypothesis that sources with MgII absorption along the lines of sight are drawn from the same distribution as objects with clean lines of sight or that objects with clean lines of sight are more strongly depolarized.

The strongest signal is achieved with $\mathrm{DP}_{\mathrm{Min} / \mathrm{Max}}$ for which the KS test yields a p-value of only $p=0.1 \%$. Figure 9 shows the distribution functions of $\mathrm{DP}_{\mathrm{Min} / \mathrm{Max}}$. As already mentioned in Section 4 , if objects at high Galactic latitude $|b|>55^{\circ}$ are excluded the signal increases to a nominal $p=0.01 \%$.

Also $\mathrm{DP}_{\text {Min/Max }}^{\prime}$ yields a strong result with $p=1.3 \%$. $\sigma_{\text {Burn }}^{\prime}$ is not as robust, due to the complex behavior of $\Pi\left(\lambda^{2}\right)$ in many sources, and for 10 objects the fit either fails to converge or yields negative $\sigma_{\text {Burn }}$ which is unphysical. Once these objects are excluded from the KS test, however, $\sigma_{\text {Burn }}^{\prime}$ yields $p=0.5 \%$.

To evaluate the real significance of the obtained pvalues (here and elsewhere in the paper) we have randomly re-scrambled objects with and without MgII absorption. The re-scrambling has been realized by randomly drawing without replacement 16 objects and declaring them artificially to be objects with clean lines of sight while the remaining 33 objects are declared as objects with interveners. Subsequently the KS test has been carried out to obtain p-values. The distribution of p-values (after 10,000 such draws) showed that the p-value estimates are conservative in the sense that $p$ - values smaller than $p=0.1 \%$ happen in $0.09 \%, p=1.3 \%$ in $0.6 \%$, and $p=0.5 \%$ in $0.2 \%$ of the realizations. This check shows that the p-values of the KS tests are conservative.

Somewhat weaker but still significant results are also achieved for $\mathrm{DP}_{\text {Half }}$ and $\sigma_{\mathrm{Burn}}$ with $p=3.9 \%$ and $p=$ $9.0 \%$. As with $\sigma_{\text {Burn }}^{\prime}$ the fit of $\sigma_{\text {Burn }}$ fails for 9 objects and those are excluded from the KS test for $\sigma_{\text {Burn }}$. For $\mathrm{DP}_{\text {Quart }}$ we see little or no effect with $p=15 \%$.

It is clear that we obtain rather different $p$-values with the six depolarization parameters and we will discuss this further below in Section 5.4. Nonetheless, altogether our results demonstrate that there is a clear and highly significant correlation between the presence of intervening MgII absorption systems and depolarization.

Farnes et al. (2015) compared fractional polarization spectral indices to the presence of MgII absorption with 41 objects and could not see any correlation between polarization structure and presence of interveners. However, recall that our sources are initially selected to be compact while the sample in Farnes et al. (2015) reduces to 15 when only flat spectrum sources are selected. Furthermore in Farnes et al. (2015) only a handful of data points along the spectrum has been available which could lead to imperfect description of the polarization structure given the complexity we can find for some sources.

\subsection{Structure in the FD distribution}

The previous section demonstrated that depolarization in the radio spectra is clearly statistically associated with the presence of intervening MgII absorption along the line of sight. Since, generically, depolarization reflects the presence of different $\phi$ coming from different parts of the source, we would then expect to see correlations between the presence of interveners and various parameters that capture the range of $\phi$ in a given source.

Armed with the quantitative parameters defined in Section 3.3, we then applied the KS tests. As before, we adopt the null hypothesis that the parameters are equally distributed between objects with and without intervening absorption systems or are enhanced for objects without interveners.

We find no significant cause to reject the null hypothesis, obtaining $p=17 \%$ for $\sigma_{\mathrm{FD}}, p=12 \%$ for $G, p=33 \%$ for $C$ and $p=24 \%$ for the subjective ranking. Figure 10 shows the distributions of the four parameters and it is fairly clear that there is no significant correlation between them and the presence of intervening absorbers. The results remain insignificant when the fractional $q$ and $u$ are used for RM Synthesis with $p=15 \%$ for $\sigma_{\mathrm{FD}}$, $p=32 \%$ for $G$ and $55 \%$ for $C$.

These null results were surprising: We clearly see the connection between interveners and depolarization in Section 5.2, but cannot then associate the complexity in the FD distribution, which we would have expected was the cause of the depolarization, with the presence of interveners. Furthermore, we actually see very little correlation between our parameterizations of FD structure and depolarization.

This result then led us to re-examine the links between the FD distribution and depolarization, to isolate that feature of the FD distribution that is most strongly 


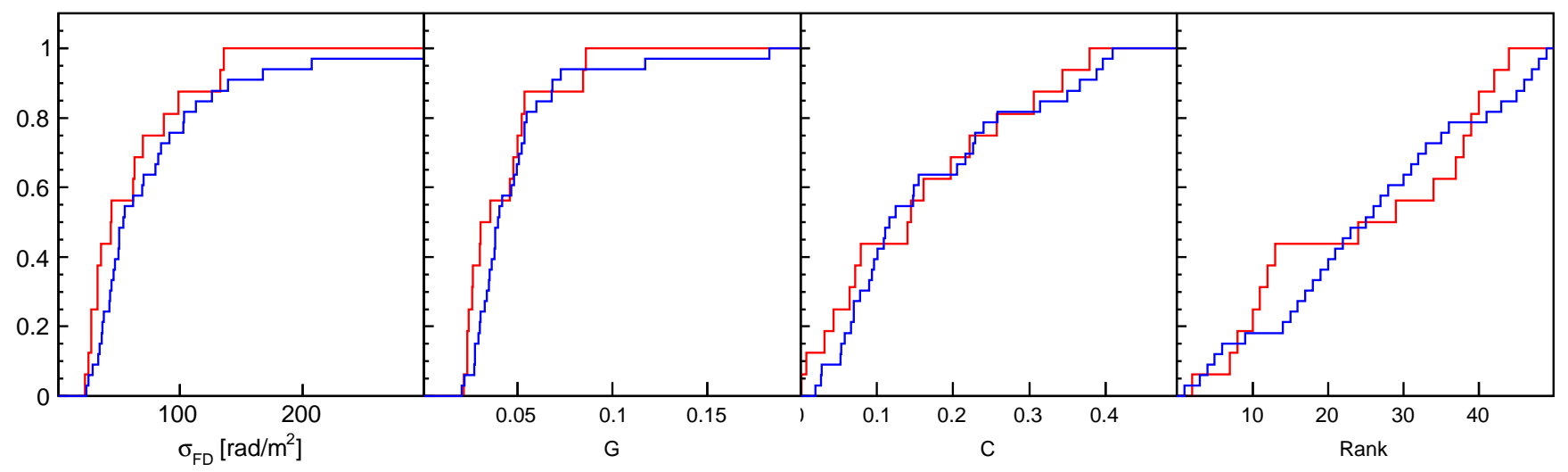

Figure 10. EDF of second moment parameter $\sigma_{\mathrm{FD}}$ (left), Gini coefficient $G$ (middle left), Coverage parameter $C$ (middle right) and subjective ranking (right) for objects without (red) and with (blue) interveners. The one-tailed KS test yields $p=32 \%, p=17 \%, p=49 \%$ and $p=24 \%$ respectively.

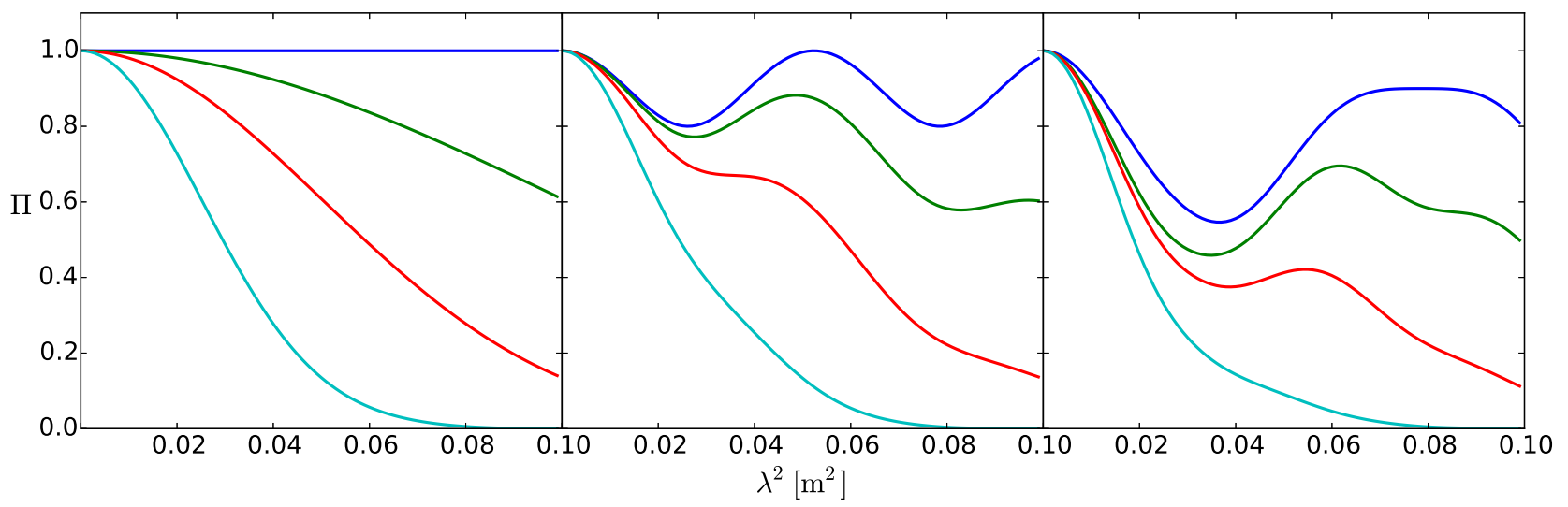

Figure 11. Obtained polarization structure $\Pi\left(\lambda^{2}\right)$ for a one component model (left), two component model with the secondary component carrying $10 \%$ of the total flux density and $40 \mathrm{rad} / \mathrm{m}^{2}$ separation from primary component (middle) and three component model with first secondary component carrying $20 \%$ of the total flux density and $40 \mathrm{rad} / \mathrm{m}^{2}$ away and the third component carrying $10 \%$ and $60 \mathrm{rad} / \mathrm{m}^{2}$ separated from the primary component (right). Each model is convolved with a Gaussian of width $\sigma_{\text {disp }}=0(\mathrm{blue}), \sigma_{\text {disp }}=5 \mathrm{rad} / \mathrm{m}^{2}$ (green), $\sigma_{\text {disp }}=10 \mathrm{rad} / \mathrm{m}^{2}$ (red) and $\sigma_{\text {disp }}=20 \mathrm{rad} / \mathrm{m}^{2}($ cyan $)$.

causing depolarization, and then to construct a further FD parameter that is, finally, clearly associated with the presence of intervening systems in our sample. This is the subject of the next two Sections 5.4 and 5.5. Furthermore, we are able to argue that the overall structure of the FD distribution examined in this section is actually likely reflecting other properties of the sources. This is briefly examined in Section 5.6.

\subsection{The connection between FD distribution and depolarization}

As commented at the end of the previous section, we found a surprising disconnect between the evident richness of structure in the FD distribution $F(\phi)$ and the presence of depolarization signatures in the overall polarization spectrum. The richness of the polarization structure was also surprising. As discussed already, we might expect to see simple monotonically decreasing polarization with increasing wavelength (for a simple Gaussian FD distribution) but instead see a very wide range of behavior (as in Column (iii) of Figures 1-3).

The polarized flux density of a given source at a given wavelength represents the vector sum of the complex representations of the different $\phi$ components, each of which rotates at a speed (in $\lambda^{2}$ space) that is proportional to $\phi$. It is worth distinguishing between the effects on $P\left(\lambda^{2}\right)$ of a few components in $F(\phi)$ that are widely separated in $\phi$, referred to as "gross structure" in the following, and the effects of very closely spaced, or continuous, structure in $F(\phi)$, as for example in the Gaussian width of a particular component, referred to as "fine structure". The former causes large variations in the polarized flux density with wavelength as the small number of polars rotate around each other producing an oscillatory behavior in the total amplitude, i.e. in the polarized flux density. In contrast, the fine structure in $\phi$ within a feature produces a more steady decrease in polarization as the continuous distribution of Faraday Rotation causes a progressive cancellation of polarized flux.

The FD distributions of many sources in Figure 1-3 show multiple discrete components, i.e. gross structure, and the parameters which we constructed in the previous section, including our own subjective ranking, were based primarily on the presence of these multiple discrete 
components rather than the width of individual components.

Further evidence for the distinction between multiple components and the widths of them (i.e. between gross and fine structure) comes from comparison of the $\sigma_{\mathrm{FD}}$ values to the parameters obtained from the depolarization curves (i.e. $\sigma_{\text {Burn }}$ or $\left.\sigma_{\text {Burn }}^{\prime}\right)$. Although we would have expected those parameters to trace the same physical quantity, i.e. the dispersion of the FD distribution, we obtain $\sigma_{\mathrm{FD}}$ to be an order of magnitude larger than the depolarization parameters. This could be explained by arguing that $\sigma_{\mathrm{FD}}$ describes gross structures while $\sigma_{\mathrm{Burn}}$ and $\sigma_{\text {Burn }}^{\prime}$ describe fine structures. The $\sigma_{\mathrm{PC}}$ parameter is sensitive to these fine structure effects because it measures the widths of the primary components. We will show later that they are of similar size as $\sigma_{\text {Burn }}$ and $\sigma_{\text {Burn }}^{\prime}$.

We explore the effects of both discrete components and the Gaussian width on the polarization $P(\lambda)$ using a simple toy model. The toy model consists of $N=1000 \mathrm{FD}$ cells which are each associated with $\phi_{i}$ according to an underlying FD distribution, assuming that all cells have the same initial phase $\psi$ and also assuming unit total flux density, $I$, so that $P(\lambda)=\Pi(\lambda)$. The polarization $P\left(\lambda^{2}\right)$ is then determined by

$$
P\left(\lambda^{2}\right)=\sqrt{U\left(\lambda^{2}\right)^{2}+Q\left(\lambda^{2}\right)^{2}}
$$

where

$$
\begin{aligned}
& U\left(\lambda^{2}\right)=\frac{1}{N} \sum_{i}^{N} \sin \left(2 \phi_{i} \lambda^{2}\right) \\
& Q\left(\lambda^{2}\right)=\frac{1}{N} \sum_{i}^{N} \cos \left(2 \phi_{i} \lambda^{2}\right) .
\end{aligned}
$$

Figure 11 shows the resulting depolarization structure of a few simple models. The left panel corresponds to a model with one $\phi$ component, the middle panel to a model with two components where the secondary component carries $10 \%$ of the total flux density. and the right panel to a model with three components where the first secondary component carries $20 \%$, and a second $10 \%$, of the total flux density. All $\phi$ components have been convolved with a Gaussian of different widths in $\phi$, namely $\sigma_{\text {disp }}=0$ (blue), $\sigma_{\text {disp }}=5 \mathrm{rad} / \mathrm{m}^{2}$ (green), $\sigma_{\text {disp }}=10 \mathrm{rad} / \mathrm{m}^{2}(\mathrm{red})$ and $\sigma_{\text {disp }}=20 \mathrm{rad} / \mathrm{m}^{2}$ (cyan), respectively.

We see that highly non-monotonic and complex polarization structure is produced by adding just a few additional components. However, the overall depolarization is mainly driven by the convolution with the Gaussian rather than by these multiple components. Multiple discrete components in contrast tend to add oscillatory features to the polarization structure. The small amplitude of the oscillations reflects the small fractions of the flux density in the secondary components. In contrast, the convolution leads to phase dispersions affecting all of the polarized flux density.

We can now return to the different parameterizations of the depolarization introduced in Section 3.2 and examine which of them best reflects the fine structure $\sigma_{\text {disp }}$, recalling that these were correlated to different degrees with the presence of MgII intervening absorption. We use the same type of simple models, but now construct 6000 models by varying the positions of the secondary component(s) relative to the primary component (between zero and $200 \mathrm{rad} / \mathrm{m}^{2}$ ), by varying the combined relative flux density contribution of the secondary component(s) (up to $40 \%$ of the total) and also by varying the initial phases $\psi$ of the secondary component(s) relative to the primary component, and to each other (between 0 and $\pi$ ). These variations reflect what we see in the FD distributions of our sample quasars (cf. $\sigma_{\mathrm{FD}}$ and $C$ in Figure 10). Furthermore each of these 6000 models is convolved with a range of $\sigma_{\text {disp }}$ between 0 and $40 \mathrm{rad} / \mathrm{m}^{2}$. The obtained DP parameters of those models are shown in the different panels of Figure 12. As with the real data, it was not always possible to fit $\sigma_{\text {Burn }}$ (bottom left) and $\sigma_{\text {Burn }}^{\prime}$ (bottom right) with physically meaningful values. The red lines in each panel shows the behavior of the single component model. As a comparison, the distribution of the corresponding DP parameters in our observed quasar sample are represented by the histograms on the righthand axis.

As discussed above, the feature in the FD distribution which most drives depolarization is $\sigma_{\text {disp }}$. Hence for our purposes a "good" DP parameter should be one which well traces $\sigma_{\text {disp. }}$. Figure 12 shows the relation of the previously defined DP parameters to $\sigma_{\text {disp }}$ for the simple one component model (red line) and as a comparison for more complex models with two or three components (black lines). It shows how the appearance of additional components can bias DP and blur the relation between $\sigma_{\text {disp }}$

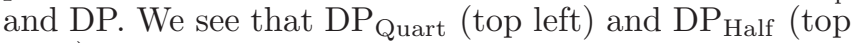
right) can be both increased and decreased by the presence of secondary components while $\mathrm{DP}_{\text {Min/Max }}$ (middle left) and $\mathrm{DP}_{\text {Min/Max }}^{\prime}$ (middle right) mostly tend to be increased.

Both $\sigma_{\text {Burn }}$ (bottom left) and $\sigma_{\text {Burn }}^{\prime}$ (bottom right) work surprisingly well in recovering an estimate of $\sigma_{\text {disp }}$, despite the difficulties of fitting the Burn model due to the non monotonic structure of $\Pi\left(\lambda^{2}\right)$. For DP $\mathrm{Quart}_{\text {and }}$ $\mathrm{DP}_{\text {Half }}$ most of the objects in our sample are in that region where secondary components can strongly affect them. As opposed to $\mathrm{DP}_{\text {Quart }}$ or $\mathrm{DP}_{\text {Half }}$, we see that for $\mathrm{DP}_{\text {Min/Max }}$ and $\mathrm{DP}_{\text {Min/Max }}^{\prime}$ a fair portion of the sample have values close to 1 , where the value is more robust against additional components.

This analysis, based on a simple toy model, therefore offers a possible explanation as to why we got different KS significances for the different DP parameters in Section 5.2. Recall that we saw the strongest correlations with the presence of intervening absorption with $\mathrm{DP}_{\text {Min/Max }}$ and $\mathrm{DP}_{\text {Min/Max }}^{\prime}$, and also, when measurable, with $\sigma_{\text {Burn }}$ and especially $\sigma_{\text {Burn }}^{\prime}$. We would have seen this behavior if the presence of MgII absorption is associated with the fine structure $\sigma_{\text {disp }}$ rather than the presence of the multiple components which dominate the visual impression of the FD distributions in Figures 1-3.

We therefore suggest that intervening material is primarily responsible for broadening the FD distribution $F(\phi)$ rather than the presence of the multiple discrete components. We will test this in the next Section 5.5 and provide a further argument in favor of this idea in the subsequent Section 5.6. 


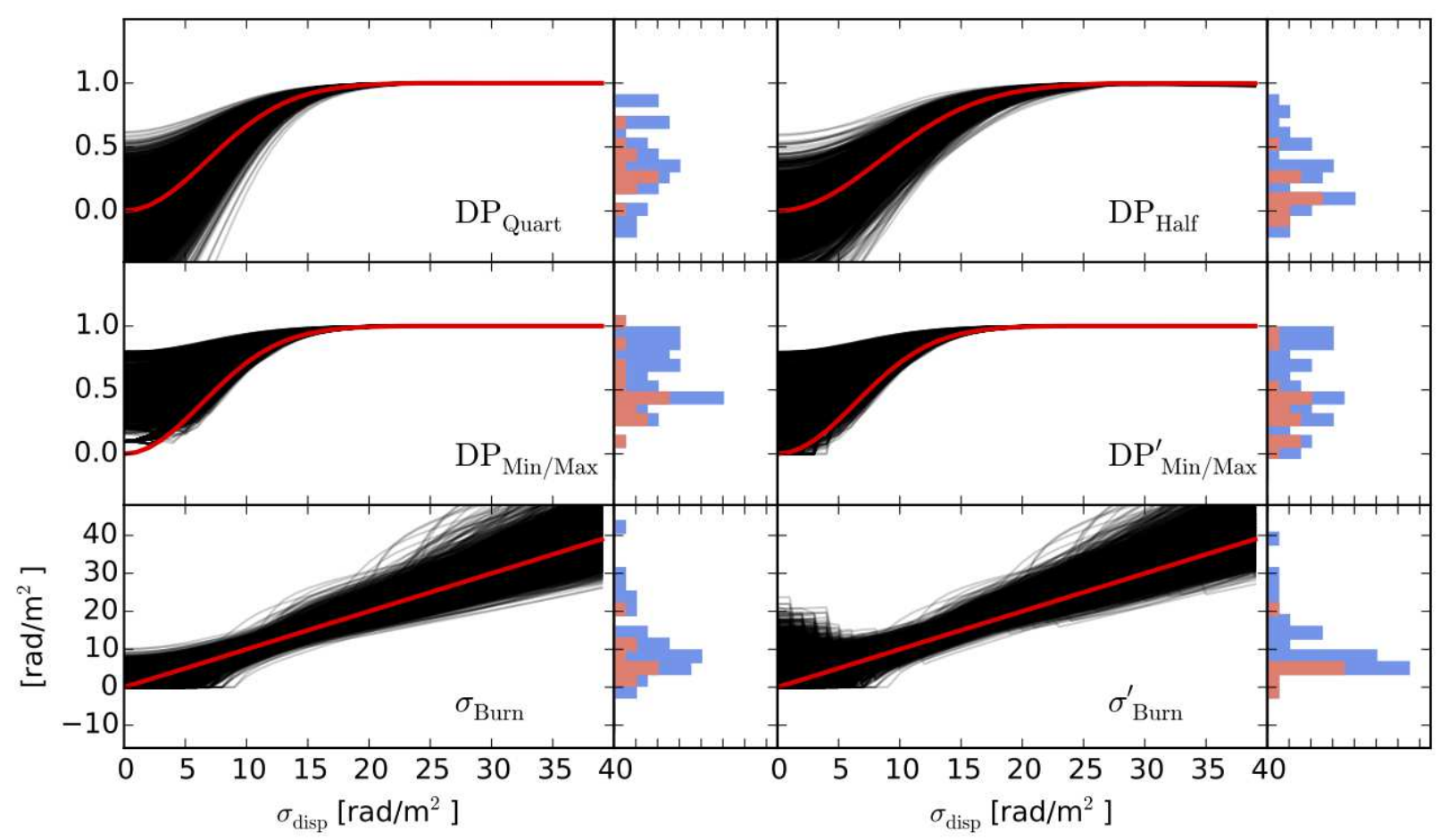

Figure 12. 6000 one, two or three component Faraday screen models have been implemented by varying relative Faraday Depth, size and initial angle of the components and each model is convolved with $\sigma_{\text {disp }}$ between 0 and $40 \mathrm{rad} / \mathrm{m}^{2}$. The obtained depolarization parameter $\mathrm{DP}_{\mathrm{Quart}}$ (top left), $\mathrm{DP}_{\text {Half }}$ (top right), $\mathrm{DP}_{\text {Min/Max }}$ (middle left), $\mathrm{DP}_{\text {Min/Max }}^{\prime}$ (middle right), $\sigma_{\text {Burn }}$ (bottom left) and $\sigma_{\text {Burn }}^{\prime}$ (bottom right) for each model is represented by the black lines. The red line represents the one component model. The histograms on the right show the respective depolarization parameter distribution of objects without (red) and with (blue) interveners in the sample. The histograms are stacked.

\subsection{Intervener Effects in Faraday Depth}

In the previous section we postulated that intervening material, traced by the MgII absorption, affects the observed FD distribution by means of a convolution effect. $\sigma_{\mathrm{PC}}$, introduced in Section 3.3, is a parameter constructed to be insensitive to the appearance of multiple components.

In Figure 13 we compare the $\sigma_{\mathrm{PC}}$ to $\sigma_{\mathrm{Burn}}^{\prime}$. The $\sigma_{\mathrm{RMSF}}$ in the VLA data is around $17 \mathrm{rad} / \mathrm{m}^{2}$ and is around 24 $\mathrm{rad} / \mathrm{m}^{2}$ for ATCA. Most objects have $\sigma_{\mathrm{PC}}$ smaller than that, i.e. those sources are barely resolved in $\phi$ space, and the values should be interpreted with some caution. Nevertheless, and despite the difficulties in obtaining fits to $\Pi\left(\lambda^{2}\right)$ with the Burn model, we see a reasonable overall correlation between $\sigma_{\mathrm{PC}}$ and $\sigma_{\mathrm{Burn}}^{\prime}$.

If we now construct our usual KS test between absorption and $\sigma_{\mathrm{PC}}$, i.e. a one-tailed test with the null hypothesis that objects with MgII absorption along the lines of sight are drawn from the same distribution of $\sigma_{\mathrm{PC}}$ as objects with clean lines of sight or that objects with clean lines of sight have enhanced $\sigma_{\mathrm{PC}}$, we can reject the null hypothesis with $p=3.5 \%$ (Figure 14). When re-scrambling objects with and without MgII absorption along the lines of sight as in Section $5.1 p=3.5 \%$ or smaller happens in $1.9 \%$ of the realizations.

To assess the significance of this result, it should be borne in mind that most of the objects were not resolved in $\phi$ space. It is noticeable that all of the quasars with $\sigma_{\mathrm{PC}}$ significantly larger than $\sigma_{\mathrm{RMSF}}$ have interven-

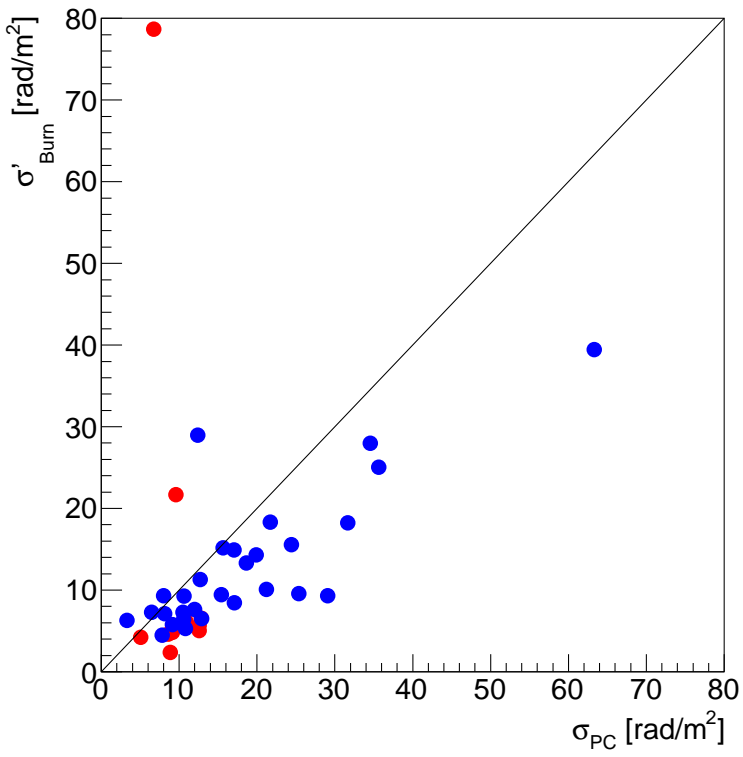

Figure 13. Comparison between the depolarization parameter $\sigma_{\text {Burn }}^{\prime}$ and the standard deviation of the primary component $\sigma_{\mathrm{PC}}$ for objects without (red) and with (blue) interveners. 10 objects for which the $\sigma_{\text {Burn }}^{\prime}$ fit failed are missing.

\section{ing MgII absorption.}

When we use the fractional $q$ and $u$ for RM Synthesis we obtain here an even more significant $\mathrm{p}$-value, with $p=$ $0.09 \%$. Sources with spectral indices different than zero, i.e. sources which vary along the observed frequency, 


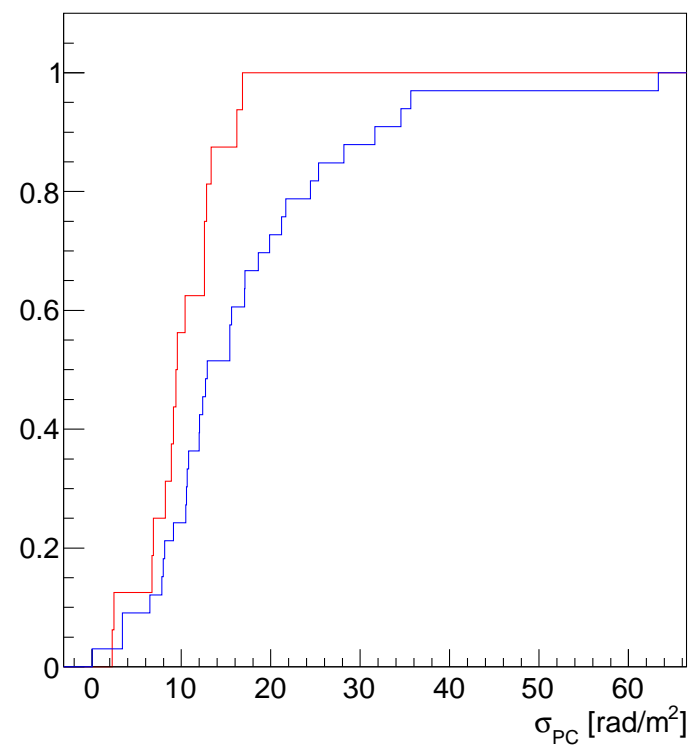

Figure 14. EDF of the depolarization parameter $\sigma_{\mathrm{PC}}$ for objects without (red) and with (blue) interveners. The one-tailed KS test yields $p=3.5 \%$.

will have associated variations also in the polarized flux density, i.e. in $Q$ and $U$, and this will affect the FD distribution. Since these variations are normally gradual with frequency, these will rather affect parameters like $\sigma_{\mathrm{PC}}$ than adding new secondary components. This then would blur the KS Test with $\sigma_{\mathrm{PC}}$. Using fractional $q$ and $u$ is an attempt to take out this effect and indeed it yields a stronger result.

The strong association of both, depolarization and $\sigma_{\mathrm{PC}}$, with intervening material indicates that intrinsic effects within the sources do not contribute much to the fine structure of the Faraday Depth within each components, i.e. $\sigma_{\text {disp }}$. This could be due to different properties of the magnetized plasma within sources compared to that in the intervening systems traced by MgII absorption. However, redshift effects should also be considered here. A given dispersion in the rest-frame $F(\phi)$ at some redshift $z$ will produce an observed $\sigma_{\text {disp }}$ that scales as $(1+z)^{-2}$. Since the quasars are generally at significantly higher redshifts than the intervening absorbing systems, this could explain why the observed $\sigma_{\text {disp }}$ is dominated by the intervening systems.

\subsection{Intrinsic effects in Faraday Depth}

We have argued above that the presence of multiple discrete components in the FD distributions $F(\phi)$ of our sources (to which the parameters we introduced in Section 5.3 were particularly sensitive) is not associated with the presence of intervening MgII absorption. In contrast, intervening material is clearly associated with the broadening of the FD distribution. This raises the possibility that the multiple components in $F(\phi)$ arise actually intrinsically to the radio sources.

That this is likely the case is indicated when we consider the initial phases $\psi$ of the components. This is shown on Figure 1-3 (Column (vi)). We always see that if a secondary component is present, then the initial phase of that secondary component is different than the initial phase of the primary component. In other words, the cusps in the radial $\mathbf{F}$ plots (in Column (vi)), that correspond to the peaks in the $F(\phi)$ plots in Column (v), lie at different azimuthal angles representing the initial phase $\psi$.

An important point is that source components with different initial phase $\psi$ must come from spatially distinct emission regions, either in the plane of the sky, or along the line of sight. This is because $\psi$ represents the phase of emission before any Faraday Rotation. It is then quite reasonable to imagine that these different emission regions have associated with them different intrinsic $\phi$. This would naturally produce the multiple $\phi$ components, each with distinct $\phi$ and $\psi$, in the overall FD distribution, as observed.

This argument above is however not watertight: It could also be imagined that different source regions (with different $\psi$ ) lying behind different parts of an intervening system would also suffer vastly different amounts of Faraday Rotation. We could for instance imagine a chequerboard intervening screen with just two values of intervening $\phi, \phi_{1}$ and $\phi_{2}$, which lies in front of a source in which $\psi$ varies spatially. We would then observe a Faraday Depth with two separated peaks $\phi_{1}$ and $\phi_{2}$, each with a $\psi$ that reflected the distribution of $\psi$ of the source regions behind the $\phi_{1}$ and $\phi_{2}$ cells of the intervening screen. This would also produce two distinct components in $F(\phi)$ with different $\psi$, with all of the Faraday Rotation coming from the intervening system. However, in this case, we would expect to see a correlation between the presence of MgII and the parameters introduced earlier that are sensitive to the presence of these multiple components in the FD distribution. This is not seen, suggesting that indeed, the different $\phi$ of the multiple components originate intrinsically to the source.

Also the fact, that we do not see a single example in which two or more cusps have the same $\psi$ which would happen e.g. if a chequer-board intervening screen as described before lies in front of a source with constant $\psi$, should be taken as an indication that distinct components in the FD distribution arise from intrinsic effects.

We conclude therefore that the gross structure complexity and multiple distinct components in $F(\phi)$ that are seen in many sources are produced by effects intrinsic to the sources, while intervening material introduces a small but pervasive broadening of the FD distribution. We explore further the implications of the latter effect in the final Discussion section immediately following. Further exploration of the properties of the background sources from QU-fitting (O'Sullivan et al. (2012), Sun et al. (2015)) and analysis of the shape of the $\mathbf{F}$ plots is beyond the scope of the current work but we plan to develop this further in the future. The results of the QUfitting will also be very interesting to be compared with $\sigma_{\mathrm{PC}}$.

\section{DISCUSSION}

In this section we will try to be more quantitative about the size of the Faraday Effects that are associated with the presence of intervening material, as revealed by MgII absorption. This will enable us to better characterize the physical properties of this material.

We estimate the dispersion of $\phi$ introduced by intervening systems. The largest separation in the $\sigma_{\mathrm{PC}}$ empirical distribution functions for sources with and without in- 
terveners is observed at $\sigma_{\mathrm{PC}}=16 \mathrm{rad} / \mathrm{m}^{2}$. As noted earlier, this value should be treated with caution because the $\phi$ resolution limit of our experiment is roughly $\sigma_{\mathrm{RMSF}}=17 \mathrm{rad} / \mathrm{m}^{2}$ for $\mathrm{VLA}$ and $\sigma_{\mathrm{RMSF}}=24 \mathrm{rad} / \mathrm{m}^{2}$ for ATCA. The difficulties of the RMSF can to a certain degree be circumvented by simply subtracting in quadrature the median $\sigma_{\mathrm{PC}}$ of sources with and without absorbers (since the RMSF has been subtracted from both $\sigma_{\text {fit }}$ equally). This yields an estimate for the excess $\sigma_{\mathrm{PC}} \approx 8.6 \mathrm{rad} / \mathrm{m}^{2}$.

The maximum separation seen in the $\sigma_{\text {Burn }}^{\prime}$ distribution is at $\sigma_{\text {Burn }}^{\prime}=7 \mathrm{rad} / \mathrm{m}^{2}$. But also here this value should be treated with caution because of the nonmonotonic structure in $\Pi\left(\lambda^{2}\right)$ and the rather ad hoc approach to choosing the wavelength range for the fit (only long-wards of the peak in polarization). Likewise, subtracting the median $\sigma_{\text {Burn }}^{\prime}$ values of the distribution with and without absorbers in quadrature gives an estimate for the excess $\sigma_{\text {Burn }}^{\prime} \approx 8 \mathrm{rad} / \mathrm{m}^{2}$.

$\sigma_{\mathrm{PC}}$ and $\sigma_{\text {Burn }}^{\prime}$ attempt to trace $\sigma_{\text {disp }}$ by different approaches. The fact that their values are similar gives some confidence that the proposed scenario is selfconsistent.

From the current work we will use a value of order $10 \mathrm{rad} / \mathrm{m}^{2}$ as a crude order of magnitude estimate of the FD dispersion that is associated with intervening systems. This is an estimate of the range of the observed $\phi$ within (i.e. across the face of) a given intervening system. To convert to the rest-frame of the absorber, we must multiply by $(1+z)^{2}$, i.e. by a factor of order 2 for our median absorber redshift of $z \approx 0.5$, yielding of order $\sigma_{\text {int, } 0} \approx 20 \mathrm{rad} / \mathrm{m}^{2}$. This value is of the same order of magnitude as the spread in Galactic RMs $\sigma_{\mathrm{MW}} \approx 8 \mathrm{rad} / \mathrm{m}^{2}$ as estimated by Schnitzeler (2010). Other works have claimed $\sigma_{\mathrm{LMC}} \approx 81 \mathrm{rad} / \mathrm{m}^{2}$ for the Large Magellanic Cloud (Gaensler et al. (2005)) and $\sigma_{\mathrm{SMC}} \approx 40 \mathrm{rad} / \mathrm{m}^{2}$ for the Small Magellanic Cloud (Mao et al. (2008)).

As in Bernet et al. (2008) we can crudely associate a $\phi$ with an estimate of magnetic field strength using an estimate of the free electron column density in the interveners based on the known MgII column density. Assuming a compact absorber system at redshift $z$ and the total magnetic field strength to be $B=\sqrt{3} B_{\|}$this reformulates equation 2 as (see Bernet et al. (2008))

$$
B=6.6 \times 10^{12} \frac{(1+z)^{2}}{N_{\mathrm{e}}} \phi
$$

where $B$ is in units of $\mathrm{G}, N_{\mathrm{e}}$ is the ion column density in units of $\mathrm{cm}^{-2}$ and $\phi$ is the Faraday Depth in units of $\mathrm{rad} / \mathrm{m}^{2}$. This estimate ignores any field reversals within the system. Moreover, assuming neutralhydrogen column density of $N(H I) \approx 10^{19} \mathrm{~cm}^{2}$ (Rao et al. (2006)) and a hydrogen ionization fraction of $\bar{x} \approx 0.90$ (Prochaska et al. (2006), Proux et al. (2007)) we estimate $N_{\mathrm{e}} \approx 9 \times 10^{19} \mathrm{~cm}^{2}$. Values of $\sigma_{\text {int }}$ of order $10 \mathrm{rad} / \mathrm{m}^{2}$ are associated with random fields of order $\sigma_{B} \approx \sqrt{m} \times 3 \mu \mathrm{G}$, where $m$ is the typical number of reversals within the intervening system along a given one-dimensional line of sight. Since the physical properties of the intervening absorption systems are barely constrained, even order of magnitude estimates of $m$ would be unreliable at this stage.

Recently Rieder \& Teyssier (2016) have investigated small-scale dynamo effects in high redshift galaxies performing magneto-hydrodynamic simulations which enables them to predict the strength and turbulence of magnetic fields in the circumgalactic medium. A detailed comparison of their predictions with our observations is beyond the scope of this paper, but will be presented elsewhere.

Finally we remark that our results emphasize that the clearest signatures of the presence of intervening material are to be seen in the dispersion of Faraday Depth $\sigma_{\text {disp }}$ within the spatial extent of a given individual source, as traced by our $\sigma_{\mathrm{PC}}$ and depolarization parameters, rather than in the overall Faraday Depth, that is traced by $\phi_{\max }$ or RM (Bernet et al. (2008)), which instead are measuring uniform effects across the whole source. This should not be surprising. Because of orientation effects, the enhancement of $\phi_{\max }$ for sources with MgII absorption lines compared to clean sources can only be made statistically, i.e. by comparing the widths of the $\phi_{\max }$ or RM distributions, while $\sigma_{\mathrm{PC}}$ as well as the depolarization parameters are a direct measure on a source by source basis. Put another way, the signal of interveners will be seen in the first moment of the $\sigma_{\text {disp }}$ distribution, but only in the second moment of the $\phi_{\max }$ or RM distribution.

We also remark on the fact that most of the $F(\phi)$ distributions are barely resolved by the RMSF of the instrumental set up, especially for the sources without intervening systems. This suggests that further improvements in this through the extension of the wavelength baseline in RM Synthesis may be useful.

\section{SUMMARY}

We have presented new continuous radio polarization measurements taken with the VLA and ATCA of 49 unresolved quasars for which we also have a census of intervening MgII absorption systems, down to $0.1 \AA$ rest frame equivalent width. We apply RM Synthesis on the radio data and compile a set of high quality $F(\phi)$ FD distributions. The $F(\phi)$ exhibit a rich diversity in structure and complexity.

In essentially all objects there is a pronounced, roughly Gaussian, primary component in $F(\phi)$. About two thirds of the sample also exhibit additional secondary components, but these display a range of structure. The overall polarization of the sources $P(\lambda)$ are also strikingly varied.

We have investigated the connections between features in the $F(\phi)$ distributions and the polarization $P(\lambda)$ and correlations between these and the presence of interveners, and reach the following main conclusions:

- We compare our completely independent radio data with the RM data used in Bernet et al. (2008) and can, in the main, confirm the reliability of the latter. However, with our substantially smaller sample we only can recover a marginal result for the connection between peak $\phi_{\max }$ and the presence of MgII absorption in the optical spectrum.

- We demonstrate, however, a strong connection between the presence of intervening absorption and the depolarization of the source, obtaining $p \approx 1 \%$ 
with a one-tailed KS test for three different parameters describing depolarization. This result firmly establishes the connection between radio properties and intervening absorption and affirms the inhomogeneous nature of the intervener screens.

- Surprisingly, given the strong association with depolarization, we do not see significant correlations between the gross structure complexity of $F(\phi)$ and the presence of interveners, or indeed with our measures of depolarization. Instead, analysis of the initial phases of the different $\phi$ components, suggests that the most visible structure in $F(\phi)$ that is caused by multiple discrete components, actually stems from effects intrinsic to the background radio sources.

- In contrast, the effect of the intervening systems is manifested by a systematic broadening of the overall $F(\phi)$. Parameters describing this broadening are correlated with the presence of intervening absorption $(p \approx 5 \%)$. Furthermore, we also show that it is this fine structure broadening of components in $F(\phi)$, rather than the presence of multiple components, that is primarily responsible for the observed depolarization, tying this result back to the strong connection with depolarization.

- We estimate that the typical effect of intervening MgII absorber systems (which generally have $0.4<z<1.4$ ) is to produce a broadening of the individual components in $F(\phi)$ of order $10 \mathrm{rad} / \mathrm{m}^{2}$. Similar estimates come from fitting models to the depolarization spectrum. This can be associated with random fields of order $\sigma_{B} \approx \sqrt{m} \times 3 \mu \mathrm{G}$, where $m$ is the number of field reversals along the line of sight.

Our new findings considerably strengthen the evidence that intervening MgII absorption systems have detectable signatures on the polarization properties of background quasars, and that these can be used to probe the magnetic field structure of these systems, which are known to be the outflow regions of star forming galaxies at intermediate redshifts.

We are grateful to the anonymous referee for their comments which drew our attention to the possible Galactic effects. We thank Philip Schmidt (MPIfR) for valuable help during the calibration, imaging and RM Synthesis of the VLA data. We also thank Aritra Basu for refereeing this report internally at MPIfR. This research has been supported by the Swiss National Science Foundation. This work was supported by the Australian Research Council through grants FL100100114 and FS100100033, and by the National Sciences and Engineering Research Council of Canada through grant RGPIN-2015-05948. The Dunlap Institute is funded through an endowment established by the David Dunlap family and the University of Toronto. SPO acknowledges support from UNAM through the PAPIIT project IA103416. National Radio
Astronomy Observatory is a facility of the National Science Foundation operated under cooperative agreement by Associated Universities, Inc. The Australia Telescope Compact Array is part of the Australia Telescope National Facility which is funded by the Australian Government for operation as a National Facility managed by CSIRO.

\section{REFERENCES}

Abraham, R., \& van Bergh, S. 2003, ApJ, 588, 218

Anderson, C. S., Gaensler, B. M., Feain, I. J., \& Franzen, T.

M. O. 2015, The Astrophysical Journal, 815, 49

Beck, R., Frick, P., Stepanov, R., \& Sokoloff, D. 2012, A\&A, 543, A113

Bernet, M., Miniati, F., \& Lilly, S. 2010, ApJ, 711, 380

-. 2012, ApJ, 761, 144

- 2013, ApJ, 772, L28

Bernet, M. L., Miniati, F., Lilly, S. J., Kronberg, P. P., \& Dessauges-Zavadsky, M. 2008, Nature, 454, 302

Bhat, P., \& Subramanian, K. 2013, MNRAS, 429, 2469

Bordoloi, R., Lilly, S., Knobel, C., et al. 2011, ApJ, 743, 10

Brentjens, M., \& de Bruyn, A. 2005, A\&A, 441, 1217

Burn, B. 1966, MNRAS, 133, 67

Clark, B. 1980, Astron. Astrophys., 89, 377

Farnes, J., Gaensler, B., \& Carretti, E. 2014, ApJS, 212, 15

Farnes, J., O'Sullivan, S., Corrigan, M., \& Gaensler, B. 2015, ApJ, 795, 63

Farnsworth, D., Rudnick, L., \& Brown, S. 2011, The

Astronomical Journal, 141, 191

Frick, P., Sokoloff, D., Stepanov, R., \& Beck, R. 2010, MNRAS, 401, L24

Gaensler, B. M., Haverkorn, M., Staveley-Smith, L., et al. 2005, Science, 307, 1610

Gardner, F., \& Whiteoak, J. 1966, ARA\&A, 4, 245

Gini, C. 1912, reprinted in Memorie di Metodologia Statistica, ed. E. Pizetti \& T. Salvemini (1955; Rome: Livreria Eredi Virgilio Veschi)

Greisen, E. 2003, Ap\&SS, 285, 109

Hammond, A. M., Robishaw, T., \& Gaensler, B. M. 2012 arXiv:1209.1438v3

Heald, G., Braun, R., \& Edmonds, R. 2009, A\&A, 503, 409

Högbom, J. 1974, A\&AS, 15, 417

Joshi, R., \& Chand, H. 2013, MNRAS, 434, 3566

Kronberg, P., Bernet, M., Miniati, F., \& Lilly, S. 2008, ApJ, 676, 70

Li, F., Cornwell, T., \& de Hoog, F. 2011, A\&A, 531, A126

Mantovani, F. Mack, K., Montenegro-Montes, F., Rossetti, A., \& Kraus, A. 2009, A\&A, 502, 61

Mao, S., Gaensler, B., \& Stanimirovi, S. 2008, ApJ, 688, 1029

McMullin, J., Waters, B., Schiebel, D., Young, W., \& Golap, K. 2007, ASP Conf. Ser., 376, 127

Oppermann, N., Junklewitz, H., Greiner, M., et al. 2015, Astronomy \& Astrophysics, 575, A118

O'Sullivan, S., Brown, S., Schnitzeler, D., et al. 2012, MNRAS, 421,3300

Perley, R., \& Butler, B. 2013, ApJ, 206, 16

Prochaska, J., O'Meara, J., Herbert-Fort, S., et al. 2006, ApJ, 648, 97

Proux, C., Dessauges-Zavadsky, M., D’Odorico, S., Kim, T., \&

McMahon, R. 2007, MNRAS, 382, 177

Rao, S., Turnshek, D., \& Nestor, D. 2006, ApJ, 636, 610

Rieder, M., \& Teyssier, R. 2016, MNRAS, 457, 1722

Rossetti, A., Dallacasa, D., Fanti, C., Fanti, R., \& Mack, K. 2008, A\&A, 487, 865

Sault, R., Teuben, P., \& Wright, M. 1995, ASP Conference Series, 77,433

Schnitzeler, D. 2010, MNRAS, 409, L99

Schnitzeler, D., \& Lee, K. 2015, MNRAS, 447, L26

Schwab, F. 1984, AJ, 89, 1076

Shukurov, A., Sokoloff, D., Subramanian, K., \& Brandenburg, A. 2006, A\&A, 448, L33

Sokoloff, D., Bykov, A., Shukurov, A., et al. 1998, MNRAS, 299 189

Sun, X. H., Rudnick, L., Akahori, T., et al. 2015, AJ, 149, 60

Taylor, A., Stil, J., \& Sunstrum, C. 2009, ApJ, 702, 1230

Tribble, P. 1991, MNRAS, 250, 726 